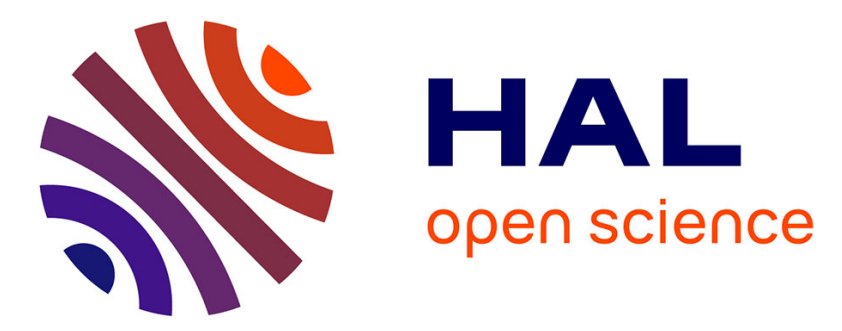

\title{
Continuum limits of nonlocal $p$-Laplacian variational problems on graphs
}

\author{
Yosra Hafiene, Jalal M. Fadili, Abderrahim Elmoataz
}

\section{To cite this version:}

Yosra Hafiene, Jalal M. Fadili, Abderrahim Elmoataz. Continuum limits of nonlocal $p$-Laplacian variational problems on graphs. SIAM Journal on Imaging Sciences, 2019. hal-02268163

\section{HAL Id: hal-02268163 \\ https://hal.science/hal-02268163}

Submitted on 20 Aug 2019

HAL is a multi-disciplinary open access archive for the deposit and dissemination of scientific research documents, whether they are published or not. The documents may come from teaching and research institutions in France or abroad, or from public or private research centers.
L'archive ouverte pluridisciplinaire HAL, est destinée au dépôt et à la diffusion de documents scientifiques de niveau recherche, publiés ou non, émanant des établissements d'enseignement et de recherche français ou étrangers, des laboratoires publics ou privés. 


\title{
Nonlocal $p$-Laplacian variational problems on graphs
}

\author{
Yosra Hafiene* Jalal M. Fadili* $\quad$ Abderrahim Elmoataz*
}

\begin{abstract}
In this paper, we study a nonlocal variational problem which consists of minimizing in $L^{2}$ the sum of a quadratic data fidelity and a regularization term corresponding to the $L^{p}$-norm of the nonlocal gradient. In particular, we study convergence of the numerical solution to a discrete version of this nonlocal variational problem to the unique solution of the continuum one. To do so, we derive an error bound and highlight the role of the initial data and the kernel governing the nonlocal interactions. When applied to variational problem on graphs, this error bound allows us to show the consistency of the discretized variational problem as the number of vertices goes to infinity. More precisely, for networks in convergent graph sequences (simple and weighted deterministic dense graphs as well as random inhomogeneous graphs), we prove convergence and provide rate of convergence of solutions for the discrete models to the solution of the continuum problem as the number of vertices grows.
\end{abstract}

Key words. Variational problems, nonlocal p-Laplacian, discrete solutions, error bounds, graph limits.

AMS subject classifications. 65N12, 65N15, 41A17, 05C80, 05C90, 49M25, 65K15.

\section{Introduction}

\subsection{Problem statement}

Let $\Omega \subset \mathbb{R}$ be a bounded domain, and without loss of generality we take $\Omega=[0,1]$, and the kernel $K$ is a symmetric, nonnegative, measurable and bounded function on $\Omega^{2}$. Denote the nonlocal gradient operator

$$
\nabla_{K}: u \in L^{2}(\Omega) \mapsto\left(K(x, y)^{1 / p}(u(y)-u(x))\right)_{(x, y) \in \Omega^{2}} \in L^{2}\left(\Omega^{2}\right) .
$$

We study the following variational problem for $p \in[1,+\infty[$

$$
\min _{u \in L^{2}(\Omega)}\left\{E_{\lambda}(u, g, K) \stackrel{\text { def }}{=} \frac{1}{2 \lambda}\|u-g\|_{L^{2}(\Omega)}^{2}+R_{p}(u, K)\right\} .
$$

The nonlocal regularizer $R_{p}$ is defined as

$$
R_{p}(u, K) \stackrel{\text { def }}{=} \begin{cases}\frac{1}{2 p} \int_{\Omega^{2}}\left|\nabla_{K} u(x, y)\right|^{p} d x d y & \text { if }|\cdot|^{p} \circ \nabla_{K} u \in L^{1}\left(\Omega^{2}\right) \\ +\infty & \text { otherwise. }\end{cases}
$$

Here $\lambda$ is a positive regularization parameter. The chief goal of this paper is to study numerical approximations of the nonlocal variational problem $\left(\mathcal{V} \mathcal{P}^{\lambda, p}\right)$, which in turn, will allow us to establish consistency estimates of the discrete counterpart of this problem on graphs.

${ }^{*}$ Normandie Univ, ENSICAEN, UNICAEN, CNRS, GREYC, France. 
Discretization of continuum models based on nonlocal regularization such as in $\left(\mathcal{V} \mathcal{P}^{\lambda, p}\right)$ has proven very effective in various tasks in signal/image/data processing, machine learning and computer vision. Such models have the advantage of better mathematical modeling, connections with physics and better geometrical approximations.

In many real-world problems where the data in practice is discrete, graphs constitute a natural structure suited to their representation. Each vertex of the graph corresponds to a datum, and the edges encode the pairwise relationships or similarities among the data. For the particular case of images, pixels (represented by nodes) have a specific organization expressed by their spatial connectivity. Therefore, a typical graph used to represent images is a grid graph. For the case of unorganized data such as point clouds, a graph can also be built by modeling neighborhood relationships between the data elements. For these practical reasons, recently, there has been a surge of interest in adapting and solving nonlocal variational problems such as $\left(\mathcal{V} \mathcal{P}^{\lambda, p}\right)$ on data which is represented by arbitrary graphs and networks. Using this framework, problems are directly expressed in a discrete setting. This way to proceed encompasses local and nonlocal methods in the same framework by using appropriate graph topologies and edge weights depending on the data structure and the task to be performed.

Thus, handling such data necessitates a discrete counterpart of $\left(\mathcal{V} \mathcal{P}^{\lambda, p}\right)$. One can intuitively propose the following problem

$$
\min _{u_{n} \in \mathbb{R}^{n}}\left\{E_{n, \lambda} \stackrel{\text { def }}{=} \frac{1}{2 \lambda n}\left\|u_{n}-g_{n}\right\|_{2}^{2}+R_{n, p}\left(u_{n}, K_{n}\right)\right\},
$$

where

$$
R_{n, p}\left(u_{n}, K_{n}\right) \stackrel{\text { def }}{=} \frac{1}{2 n^{2} p} \sum_{i, j=1}^{n} K_{n i j}\left|u_{n j}-u_{n i}\right|^{p} .
$$

Since the discrete nonlocal problem $\left(\mathcal{V}^{\lambda, p}\right)$ was only intuitively inspired as a numerical approximation to $\left(\mathcal{V} \mathcal{P}^{\lambda, p}\right)$, several legitimate questions then arise:

(Q.1) Is there any continuum limit to the (unique) minimizer $u_{n}^{\star}$ of $\left(\mathcal{V} \mathcal{P}_{n}^{\lambda, p}\right)$ as $n \rightarrow+\infty$ ? If yes, in what sense ? The graphs considered in $\left(\mathcal{V P}_{n}^{\lambda, p}\right)$ are rather general and it is not obvious at first glance what a continuum model should be.

(Q.2) What is the rate of convergence to this limit and what is its relation to the (unique) minimizer $u^{\star}$ of $\left(\mathcal{V} \mathcal{P}^{\lambda, p}\right) ?$

(Q.3) What are the parameters involved in this convergence rate (in particular the interplay between $n, p$ and properties of the graph), and what is their influence in the corresponding rate ?

(Q.4) Can this continuum limit help us get better insight into discrete models/algorithms and how they may be improved?

It is our primary motivation to answer these questions rigorously. Our perspective stands at the interface of numerical analysis and data processing.

\subsection{Contributions}

In this work we focus on studying the consistency of $\left(\mathcal{V} \mathcal{P}^{\lambda, p}\right)$ in which we investigate functionals with a nonlocal regularization term corresponding to the $p$-Laplacian operator. We first give 
a general error estimate in $L^{2}(\Omega)$ controlling the error between the continuum extension of the numerical solution $u_{n}^{\star}$ to the discrete variational problem $\left(\mathcal{V} \mathcal{P}_{n}^{\lambda, p}\right)$ and its continuum analogue $u^{\star}$ of $\left(\mathcal{V} \mathcal{P}^{\lambda, p}\right)$. The dependence of the bound on the error induced by discretizing the kernel $K$ and the initial data $g$ is made explicit. Under very mild conditions on $K$ and $g$, typically belonging to a large class of Lipschitz functional spaces (see Section 2.3 for details on these spaces), convergence rates can be exhibited by plugging Lemma 4.1 and Lemma 2.2 into the bounds of Theorem 4.1. It is worth pointint out that at this stage, though we have focused on the $1 \mathrm{D}$ case, these results hold true if $\Omega$ is any bounded domain in $\mathbb{R}^{d}$. This would allow for instance the application of our results to a variety of situations, typically image or point cloud processing (see Section 7 for a detailed example). Here the nodes/vertices of the graph are the pixels/points locations, and the edge weights model the neighborhood relationships. The neighbourhood can be thought of as spatial or also include value similarity such as in patch-based methods.

Secondly, we apply these results, using the theory graph limits (for instance graphons), to dynamical networks on simple and weighted dense graphs to show that the approximation of minimizers of the discrete problems on simple and weighted graph sequences converge to those of the continuum problem. This sets the question that solving a discrete variational problem on graphs has indeed a continuum limit. Under very mild conditions on $K$ and $g$, typically belonging to Lipschitz functional spaces, precise convergence rates can be exhibited. These functional spaces allow one to cover a large class of graphs (through $K$ ) and initial data $g$, including those functions of bounded variation. For simple graph sequences, we also show how the accuracy of the approximation depends on the regularity of the boundary of the support of the graph limit.

Finally, building upon these error estimates, we study networks on random inhomogeneous graphs. We combine them with sharp deviation inequalities to establish nonasymptotic convergence claims and give the rate of convergence of the discrete solution to its continuum limit with high probability under the same assumptions on the kernel $K$ and the initial data $g$.

\subsection{Relation to prior work}

Graph-based regularization in machine learning Semi-supervised learning with a weighted graph to capture the geometry of the unlabelled data and graph Laplacian-based regularization is now popular; see [36] for a review. Pointwise and/or spectral convergence of graph Laplacians were studied by several authors including $[6,32,31,16,43,53,50,44]$.

Graph-based $p$-Laplacian regularization has also found applications in semi-supervised learning such as clustering; see e.g. [57, 20, 13]. For instance, the authors of [19] obtained iterated pointwise convergence of rescaled graph $p$-Laplacian energies to the continuum (local) $p$-Laplacian as the fraction of labelled to unlabelled points is vanishingly small. The authors in [25] studied the consistency of rescaled total variation minimization on random point clouds in $\mathbb{R}^{d}$ for a clustering application. They considered the total variation on graphs with a radially symmetric and rescaled kernel $K(x, y)=\varepsilon^{-d} J(|x-y| / \varepsilon), \varepsilon>0$. This corresponds to an instance of $R_{n, p}$ for $d=1$ and $p=1$. Under some assumptions on $J$, and for an appropriate scaling of $\varepsilon$ with respect to $n$, which makes the method become localised in the large data limit, they proved that the discrete total variation on graphs $\Gamma$-converges in an appropriate topology, as $n \rightarrow \infty$, to weighted local total variation, where the weight function is the density of the point cloud distribution. Motivated by the work of [19], the authors of [45] studied consistency of the graph $p$-Laplacian for semi-supervised learning in $\mathbb{R}^{d}$. They considered both constrained and penalized minimization of $R_{n, p}$ with a radially symmetric and rescaled kernel as explained before. They uncovered regimes of $p$ and ranges on the scaling of $\varepsilon$ 
with respect to $n$ for the asymptotic consistency (in the sense of $\Gamma$-convergence) to hold. Continuing along the lines of [19], the work of [14] studies the consistency of Lipschitz semi-supervised learning (i.e., $p \rightarrow \infty$ ) on graphs in the same asymptotic limit. This work proves that Lipschitz learning is well-posed and showed that the learned functions converge to the solution of an $\infty$-Laplace type equation, depending on the choice of weights in the graph.

The Ginzburg-Landau functional has been adapted in [7] to weighted graphs in an application to machine learning and data clustering. In [26], the authors study $\Gamma$-convergence of the graph based Ginzburg-Landau functional, both the limit for zero diffusive interface parameter or when the number of graph vertices increases.

However, all the above results are asymptotic and do not provide any error estimates for finite $n$.

Nonlocal and graph-based regularization in imaging Several edge-aware filtering schemes have been proposed in the literature [56, 46, 51, 47]. The nonlocal means filter [12] averages pixels that can be arbitrary far away, using a similarity measure based on distance between patches. As shown in $[48,40]$, these filters can also be interpreted within the variational framework with nonlocal regularization functionals. They correspond to one step of gradient descent on $\left(\mathcal{V P}{ }_{n}^{\lambda, p}\right)$ with $p=2$, where $K_{n i j}=J\left(\left|x_{i}-x_{j}\right|\right)$ is computed from the noisy input image $g$ using either a distance between the pixels $x_{i}$ and $x_{j}[56,51,47]$ or a distance between the patches around $x_{i}$ and $x_{j}[12,49]$. This nonlocal variational denoising can be related to sparsity in an adapted basis of eigenvector of the nonlocal diffusion operator [17, 49, 40]. This nonlocal variational framework was also extended to handle several linear inverse problems [48, 27, 11, 28, 20, 21]. In [41, 22, 55], the authors proposed a variational framework with nonlocal regularizers on graphs to solve linear inverse problems in imaging where both the image to recover and the graph structure are inferred.

Consistency of the ROF model For local variational problems, the only work on consistency with error bounds that we are aware of is the one of [54] who studied the numerical approximation of the Rudin-Osher-Fatemi (ROF) model, which amounts to minimizing in $L^{2}\left(\Omega^{2}\right)$ the well-known energy functional

$$
E(v) \stackrel{\text { def }}{=} \frac{1}{2 \lambda}\|u-g\|_{L^{2}\left(\Omega^{2}\right)}^{2}+\|v\|_{\mathrm{TV}\left(\Omega^{2}\right)}
$$

where $g \in L^{2}\left(\Omega^{2}\right)$, and $\|\cdot\|_{\mathrm{TV}\left(\Omega^{2}\right)}$ denotes the total variation seminorm. They bound the difference between the continuum solution and the solutions to various finite-difference approximations (including the upwind scheme) to this model. They gave an error estimate in $L^{2}\left(\Omega^{2}\right)$ of the difference between these two solutions and showed that it scales as $n^{-\frac{s}{2(s+1)}}$, where $\left.\left.s \in\right] 0,1\right]$ is the smoothness parameter of the Lipschitz space containing $g$.

However, to the best of our knowledge, there is no such error bounds in the nonlocal variational setting. In particular, the problem of the continuum limit and consistency of $\left(\mathcal{V} \mathcal{P}_{n}^{\lambda, p}\right)$ with error estimates is still open in the literature. It is our aim in this work to rigorously settle this question.

\subsection{Paper organisation}

The rest of this paper is organized as follows. Section 2 collects some notations and preliminaries that we will need in our exposition. In Section 3 we briefly discuss well-posedness of 
problems $\left(\mathcal{V} \mathcal{P}^{\lambda, p}\right)$ and $\left(\mathcal{V} \mathcal{P}_{n}^{\lambda, p}\right)$ and recall some properties of the corresponding minimizers. Section 4 is devoted to the main result of the paper (Theorem 4.1) in which we give a bound on the $L^{2}$-norm of the difference between the unique minimizers of $\left(\mathcal{V} \mathcal{P}^{\lambda, p}\right)$ and $\left(\mathcal{V} \mathcal{P}_{n}^{\lambda, p}\right)$. In this section, we also state a key regularity result on the minimizer $u^{\star}$ of $\left(\mathcal{V} \mathcal{P}^{\lambda, p}\right)$. This result is then used to study networks on deterministic dense graph sequences in Section 5. First we deal with networks in simple graphs, and show in Corollary 5.1 the influence of the regularity of the boundary of the support of the graphon on the convergence rate. Secondly, in Section 5.2 we study networks on weighted graphs. Section 6 deals with networks on random inhomogeneous graphs. We quantify the rate of convergence with high probability. Numerical results are finally reported in Section 7 to illustrate our theoretical findings.

\section{Notations and preliminaries}

To provide a self-contained exposition, we will recall two key frameworks our work relies on. The first is the limit graph theory which is the notion of convergence for graph sequences developed for the analysis of networks on graphs. The second is that of Lipschitz spaces that will be instrumental to quantify the rate of convergence in our error bounds.

\subsection{Projector and injector}

Let $n \in \mathbb{N}^{*}$, and divide $\Omega$ into $n$ intervals

$$
\Omega_{1}^{(n)}=\left[0, \frac{1}{n}\left[, \Omega_{2}^{(n)}=\left[\frac{1}{n}, \frac{2}{n}\left[, \ldots, \Omega_{j}^{(n)}=\left[\frac{j-1}{n}, \frac{j}{n}\left[, \ldots, \Omega_{n}^{(n)}=\left[\frac{n-1}{n}, 1[,\right.\right.\right.\right.\right.\right.\right.
$$

and let $\mathcal{Q}_{n}$ denote the partition of $\Omega, \mathcal{Q}_{n}=\left\{\Omega_{i}^{(n)}, i \in[n] \stackrel{\text { def }}{=}\{1, \cdots, n\}\right\}$. Denote $\Omega_{i j}^{(n)} \stackrel{\text { def }}{=} \Omega_{i}^{(n)} \times \Omega_{j}^{(n)}$. Without loss of generality, we assume that the points are equispaced so that $\left|\Omega_{i}^{(n)}\right|=1 / n$, where $\left|\Omega_{i}^{(n)}\right|$ is the measure of $\Omega_{i}^{(n)}$. The discussion can be easily extended to non-equispaced points by appropriate normalization; see Section 6.

We also consider the operator $P_{n}: L^{1}(\Omega) \rightarrow \mathbb{R}^{n}$

$$
\left(P_{n} v\right)_{i} \stackrel{\text { def }}{=} \frac{1}{\left|\Omega_{i}^{(n)}\right|} \int_{\Omega_{i}^{(n)}} v(x) d x .
$$

This operator can be also seen as a piecewise constant projector of $u$ on the space of discrete functions. For simplicity, and with a slight abuse of notation, we keep the same notation for the projector $P_{n}: L^{1}\left(\Omega^{2}\right) \rightarrow \mathbb{R}^{n \times n}$.

We assume that the discrete initial data $g_{n}$ and the discrete kernel $K_{n}$ are constructed as

$$
g_{n}=P_{n} g \stackrel{\text { def }}{=}\left(g_{n 1}, \cdots, g_{n n}\right)^{\top} \text { and } K_{n}=P_{n} K \stackrel{\text { def }}{=}\left(K_{n i j}\right)_{1 \leq i, j \leq n},
$$

where

$$
g_{n i}=\left(P_{n} g\right)_{i}=\frac{1}{\left|\Omega_{i}^{(n)}\right|} \int_{\Omega_{i}^{(n)}} g(x) d x \text { and } K_{n i j}=\left(P_{n} K\right)_{i j}=\frac{1}{\left|\Omega_{i j}^{(n)}\right|} \int_{\Omega_{i j}^{(n)}} K(x, y) d x d y .
$$

Our aim is to study the relationship between the minimizer $u^{\star}$ of $E_{\lambda}(\cdot, g, K)$ and the discrete minimizer $u_{n}^{\star}$ of $E_{n, \lambda}\left(\cdot, g_{n}, K_{n}\right)$ and estimate the error between solutions of discrete approximations 
and the solution of the continuum model. But the solution of problem $\left(\mathcal{V P}{ }_{n}^{\lambda, p}\right)$ being discrete, it is convenient to introduce an intermediate model which is the continuum extension of the discrete solution. Towards this goal, we consider the piecewise constant injector $I_{n}$ of the discrete functions $u_{n}^{\star}$ and $g_{n}$ into $L^{2}(\Omega)$, and of $K_{n}$ into $L^{\infty}\left(\Omega^{2}\right)$, respectively. This injector $I_{n}$ is defined as

$$
\begin{aligned}
& I_{n} u_{n}(x) \stackrel{\text { def }}{=} \sum_{i=1}^{n} u_{n i} \chi_{\Omega_{i}^{(n)}}(x), \\
& I_{n} g_{n}(x) \stackrel{\text { def }}{=} \sum_{i=1}^{n} g_{n i} \chi_{\Omega_{i}^{(n)}}(x), \\
& I_{n} K_{n}(x, y) \stackrel{\text { def }}{=} \sum_{i=1}^{n} \sum_{j=1}^{n} K_{n i j} \chi_{\Omega_{i}^{(n)} \times \Omega_{j}^{(n)}}(x, y),
\end{aligned}
$$

where we recall that $\chi_{\mathcal{C}}$ is the characteristic function of the set $\mathcal{C}$, i.e., takes 0 on $\mathcal{C}$ and 1 otherwise.

With these definitions, we have the following well-known properties whose proofs are immediate. We define the $\|\cdot\|_{q, n}$ norm, for a given vector $u=\left(u_{1}, \cdots, u_{n}\right)^{\top} \in \mathbb{R}^{n}, q \in[1,+\infty[$,

$$
\|u\|_{q, n}=\left(\frac{1}{n} \sum_{i=1}^{n}\left|u_{i}\right|^{q}\right)^{\frac{1}{q}}
$$

with the usual adaptation for $q=+\infty$.

Lemma 2.1. For a function $v \in L^{q}(\Omega), q \in[1,+\infty]$, we have

$$
\left\|P_{n} v\right\|_{q, n} \leq\|v\|_{L^{q}(\Omega)}
$$

and for $v_{n} \in \mathbb{R}^{n}$

$$
\left\|I_{n} v_{n}\right\|_{L^{q}(\Omega)}=\left\|v_{n}\right\|_{q, n}
$$

In turn

$$
\left\|I_{n} P_{n} v\right\|_{L^{q}(\Omega)} \leq\|v\|_{L^{q}(\Omega)} .
$$

It is immediate to see that the composition of the operators $I_{n}$ and $P_{n}$ yields the operator $\operatorname{proj}_{V_{n}}=I_{n} P_{n}$ which is the orthogonal projector on the subspace $V_{n} \stackrel{\text { def }}{=} \operatorname{Span}\left\{\chi_{\Omega_{i}^{(n)}}: i \in[n]\right\}$ of $L^{1}(\Omega)$.

\subsection{Graph limit theory}

We now briefly review some definitions and results from the theory of graph limits that we will need later since it is the key of our study of the discrete counterpart of the problem $\left(\mathcal{V} \mathcal{P}^{\lambda, p}\right)$ on dense deterministic graphs. We follow considerably $[9,34]$, in which much more details can be found.

An undirected graph $G=(V(G), E(G))$, where $V(G)$ stands for the set of nodes and $E(G) \subset$ $V(G) \times V(G)$ denotes the edges set, without loops and parallel edges is called simple.

Let $G_{n}=\left(V\left(G_{n}\right), E\left(G_{n}\right)\right), n \in \mathbb{N}^{*}$, be a sequence of dense, finite, and simple graphs, i.e; $\left|E\left(G_{n}\right)\right|=O\left(\left|V\left(G_{n}\right)\right|^{2}\right)$, where |.| now denotes the cardinality of a set. 
For two simple graphs $F$ and $G$, $\operatorname{hom}(F, G)$ indicates the number of homomorphisms (adjacencypreserving maps) from $V(F)$ to $V(G)$. Then, it is worthwhile to normalize the homomorphism numbers and consider the homomorphism densities

$$
t(F, G)=\frac{\operatorname{hom}(F, G)}{|V(G)|^{|V(F)|}} .
$$

(Thus $t(F, G)$ is the probability that a random map of $V(F)$ into $V(G)$ is a homomorphism).

Definition 2.1. (cf.[34]) The sequence of graphs $\left\{G_{n}\right\}_{n \in \mathbb{N}^{*}}$ is called convergent if $t\left(F, G_{n}\right)$ is convergent for every simple graph $F$.

Convergent graph sequences have a limit object, which can be represented as a measurable symmetric function $K: \Omega^{2} \rightarrow \Omega$, here $\Omega$ stands for [0,1]. Such functions are called graphons.

Let $\mathcal{K}$ denote the space of all bounded measurable functions $K: \Omega^{2} \rightarrow \mathbb{R}$ such that $K(x, y)=$ $K(y, x)$ for all $x, y \in[0,1]$. We also define $\mathcal{K}_{0}=\{K \in \mathcal{K}: 0 \leq K \leq 1\}$ the set of all graphons.

Proposition 2.1 ([9, Theorem 2.1]). For every convergent sequence of simple graphs, there is $K \in \mathcal{K}_{0}$ such that

$$
t\left(F, G_{n}\right) \rightarrow t(F, K) \stackrel{\text { def }}{=} \int_{\Omega^{|V(F)|}} \prod_{(i, j) \in E(F)} K\left(x_{i}, x_{j}\right) d x
$$

for every simple graph $F$. Moreover, for every $K \in \mathcal{K}_{0}$, there is a sequence of graphs $\left\{G_{n}\right\}_{n \in \mathbb{N}^{*}}$ satisfying (9).

Graphon $K$ in (9) which is uniquely determined up to measure-preserving transformations, is the limit of the convergent sequence $\left\{G_{n}\right\}_{n \in \mathbb{N}^{*}}$. Indeed, every finite simple graph $G_{n}$ such that $V\left(G_{n}\right)=[n]$ can be represented by a function $K_{G_{n}} \in \mathcal{K}_{0}$

$$
K_{G_{n}}(x, y)= \begin{cases}1 & \text { if }(i, j) \in E\left(G_{n}\right) \quad \text { and } \quad(x, y) \in \Omega_{i j}^{(n)}, \\ 0 & \text { otherwise }\end{cases}
$$

Hence, geometrically, the graphon $K$ can be interpreted as the limit of $K_{G_{n}}$ for the standard distance (called the cut-distance), see [9, Theorem 2.3]. An interesting consequence of this interpretation is that the space of graphs $G_{n}$, or equivalently pixel kernels $K_{G_{n}}$, is not closed under the cut distance. The space of graphons (larger than the space of graphs) defines the completion of this space.

\subsection{Lipschitz spaces}

We introduce the Lipschitz spaces $\operatorname{Lip}\left(s, L^{q}\left(\Omega^{d}\right)\right)$, for $d \in\{1,2\}, q \in[1,+\infty]$, which contain functions with, roughly speaking, $s$ "derivatives" in $L^{q}\left(\Omega^{d}\right)$ [18, Ch. 2, Section 9].

Definition 2.2. For $F \in L^{q}\left(\Omega^{d}\right), q \in[1,+\infty]$, we define the (first-order) $L^{q}\left(\Omega^{d}\right)$ modulus of smoothness by

$$
\omega(F, h)_{q} \stackrel{\text { def }}{=} \sup _{\boldsymbol{z} \in \mathbb{R}^{d},|\boldsymbol{z}|<h}\left(\int_{\boldsymbol{x}, \boldsymbol{x}+\boldsymbol{z} \in \Omega^{d}}|F(\boldsymbol{x}+\boldsymbol{z})-F(\boldsymbol{x})|^{q} d \boldsymbol{x}\right)^{1 / q} .
$$

The Lipschitz spaces $\operatorname{Lip}\left(s, L^{q}\left(\Omega^{d}\right)\right)$ consist of all functions $F$ for which

$$
|F|_{\operatorname{Lip}\left(s, L^{q}\left(\Omega^{d}\right)\right)} \stackrel{\text { def }}{=} \sup _{h>0} h^{-s} \omega(F, h)_{q}<+\infty .
$$


We restrict ourselves to values $s \in] 0,1]$ since for $s>1$, only constant functions are in $\operatorname{Lip}\left(s, L^{q}\left(\Omega^{d}\right)\right)$. It is easy to see that $|F|_{\operatorname{Lip}\left(s, L^{q}\left(\Omega^{d}\right)\right)}$ is a semi-norm. $\operatorname{Lip}\left(s, L^{q}\left(\Omega^{d}\right)\right)$ is endowed with the norm

$$
\|F\|_{\operatorname{Lip}\left(s, L^{q}\left(\Omega^{2}\right)\right)} \stackrel{\text { def }}{=}\|F\|_{L^{q}\left(\Omega^{2}\right)}+|F|_{\operatorname{Lip}\left(s, L^{q}\left(\Omega^{d}\right)\right)} .
$$

The space $\operatorname{Lip}\left(s, L^{q}\left(\Omega^{2}\right)\right)$ is the Besov space $\mathbf{B}_{q, \infty}^{s}[18$, Ch. 2, Section 10] which are very popular in approximation theory. In particular, $\operatorname{Lip}\left(s, L^{1 / s}\left(\Omega^{d}\right)\right)$ contains the space $\mathrm{BV}\left(\Omega^{d}\right)$ of functions of bounded variation on $\Omega^{d}$, i.e. the set of functions $F \in L^{1}\left(\Omega^{d}\right)$ such that their variation is finite:

$$
V_{\Omega^{2}}(F) \stackrel{\text { def }}{=} \sup _{h>0} h^{-1} \sum_{i=1}^{d} \int_{\Omega^{d}}\left|F\left(\boldsymbol{x}+h e_{i}\right)-F(\boldsymbol{x})\right| d \boldsymbol{x}<+\infty,
$$

where $e_{i}, i \in\{1, d\}$ are the coordinate vectors in $\mathbb{R}^{d}$; see [18, Ch. 2, Lemma 9.2]. Thus Lipschitz spaces are rich enough to contain functions with both discontinuities and fractal structure.

Let us define the piecewise constant approximation of a function $F \in L^{q}\left(\Omega^{2}\right)$ (a similar reasoning holds of course on $\Omega$ ) on a partition of $\Omega^{2}$ into cells

$$
\left.\left.\left.\left.\Omega_{n i j} \stackrel{\text { def }}{=}\{] x_{i-1}, x_{i}\right] \times\right] y_{j-1}, y_{j}\right]:(i, j) \in[n]^{2}\right\}
$$

of maximal mesh size $\delta(n) \stackrel{\text { def }}{=} \max _{(i, j) \in[n]^{2}} \max \left(\left|x_{i}-x_{i-1}\right|,\left|y_{j}-y_{j-1}\right|\right)$,

$$
F_{n}(x, y) \stackrel{\text { def }}{=} \sum_{i, j=1}^{n} F_{n i j} \chi_{\Omega_{n i j}}(x, y), \quad F_{n i j}=\frac{1}{\left|\Omega_{n i j}\right|} \int_{\Omega_{n i j}} F(x, y) d x d y .
$$

One may have recognized in these expressions non-equispaced versions of the projector and injector defined above.

We now state the following error bounds whose proofs use standard arguments from approximation theory; see [30, Section 6.2.1] for details.

Lemma 2.2. There exists a positive constant $C_{s}$, depending only on $s$, such that for all $F \in$ $\left.\left.\operatorname{Lip}\left(s, L^{q}\left(\Omega^{d}\right)\right), d \in\{1,2\}, s \in\right] 0,1\right], q \in[1,+\infty]$,

$$
\left\|F-F_{n}\right\|_{L^{q}\left(\Omega^{d}\right)} \leq C_{S} \delta(n)^{s}|F|_{\operatorname{Lip}\left(s, L^{q}\left(\Omega^{d}\right)\right)} .
$$

Let $p \in] 1,+\infty\left[\right.$. If, in addition, $F \in L^{\infty}\left(\Omega^{d}\right)$, then

$$
\left\|F-F_{n}\right\|_{L^{p}\left(\Omega^{d}\right)} \leq C\left(p, q, s,\|F\|_{L^{\infty}\left(\Omega^{d}\right)}\right) \delta(n)^{s \min (1, q / p)} .
$$

where $C\left(p, q, s,\|F\|_{L^{\infty}\left(\Omega^{d}\right)}\right)$ is a positive constant depending only on $p, q, s$ and $\|F\|_{L^{\infty}\left(\Omega^{d}\right)}$.

\section{$3 \quad$ Well posedness}

We start by proving existence and uniqueness of the minimizer for $\left(\mathcal{V} \mathcal{P}^{\lambda, p}\right)$ and $\left(\mathcal{V} \mathcal{P}_{n}^{\lambda, p}\right)$. 
Theorem 3.1. Suppose that $p \in\left[1,+\infty\left[, K\right.\right.$ is a nonnegative measurable mapping, and $g \in L^{2}(\Omega)$. Then, $E_{\lambda}(\cdot, g, K)$ has a unique minimizer in

$$
\left\{u \in L^{2}(\Omega): R_{p}(u, K) \leq(2 \lambda)^{-1}\|g\|_{L^{2}(\Omega)}^{2}\right\}
$$

and $E_{n, \lambda}\left(\cdot, g_{n}, K_{n}\right)$ has a unique minimizer.

Proof : The arguments are standard (coercivity, lower semicontinuity and strict convexity) but we provide a self-contained proof (only for $E_{\lambda}(\cdot, g, K)$ ). First observe that from [3, Proposition 9.32 and Proposition 9.5], we infer that $R_{p}(\cdot, K)$ in (1) is proper convex and lower semicontinuous. Let $\left\{u_{k}^{\star}\right\}_{k \in \mathbb{N}}$ be a minimizing sequence in $L^{2}(\Omega)$. By optimality and Jensen's inequality, we have

$$
\begin{aligned}
\left\|u_{k}^{\star}\right\|_{L^{2}(\Omega)}^{2} \leq 2\left(2 \lambda E_{\lambda}\left(u_{k}^{\star}, g, K\right)+\|g\|_{L^{2}(\Omega)}^{2}\right) & \leq 2\left(2 \lambda E_{\lambda}(0, g, K)+\|g\|_{L^{2}(\Omega)}^{2}\right) \\
& =4\|g\|_{L^{2}(\Omega)}^{2}<+\infty .
\end{aligned}
$$

Moreover

$$
R_{p}\left(u_{k}^{\star}, K\right) \leq E_{\lambda}\left(u_{k}^{\star}, g, K\right) \leq E_{\lambda}(0, g, K)=\frac{1}{2 \lambda}\|g\|_{L^{2}(\Omega)}^{2}<+\infty .
$$

Thus $\left\|u_{k}^{\star}\right\|_{L^{2}(\Omega)}$ is bounded uniformly in $k$ so that the Banach-Alaoglu theorem for $L^{2}(\Omega)$ and compactness provide a weakly convergent subsequence (not relabelled) with a limit $\bar{u} \in L^{2}(\Omega)$. By lower semicontinuity of the $L^{2}(\Omega)$ norm and that of $R_{p}(\cdot, K), \bar{u}$ must be a minimizer. The uniqueness follows from strict convexity of $\|\cdot\|_{L^{2}(\Omega)}^{2}$ and convexity of $R_{p}(\cdot, K)$.

Remark 3.1. Theorem 3.1 can be extended to linear inverse problems where the data fidelity in $E_{\lambda}(0, g, K)$ is replaced by $\|g-\mathrm{A} u\|_{L^{2}(\Sigma)}^{2}$, and where $\mathrm{A}$ is a continuous linear operator. The case where $\mathrm{A}: L^{2}(\Omega) \rightarrow L^{2}(\Sigma)$ is injective is immediate. The general case is more intricate and would necessitate appropriate assumptions on $\mathrm{A}$ and a Poincaré-type inequality. For instance, if $\mathrm{A}: L^{p}(\Omega) \rightarrow L^{2}(\Sigma)$, and the kernel of A intersects constant functions trivially, then using the Poincaré inequality in [1, Proposition 6.19], one can show existence and uniqueness in $L^{p}(\Omega)$, and thus in $L^{2}(\Omega)$ if $p \geq 2$. We omit the details here as this is beyond the scope of the paper.

We now turn to provide useful characterization of the minimizers $u^{\star}$ and $u_{n}^{\star}$. We stress that the minimization problem $\left(\mathcal{V} \mathcal{P}^{\lambda, p}\right)$ that we deal with is considered over $L^{2}(\Omega)\left(L^{2}(\Omega) \subset L^{p}(\Omega)\right.$ only for $p \in[1,2]$ ) over which the function $R_{p}(\cdot, K)$ may not be finite (see $(1)$ ). In correspondence, we will consider the subdifferential of the proper lower semicontinuous convex function $R_{p}(\cdot, K)$ on $L^{2}(\Omega)$ defined as

$$
\partial R_{p}(u, K) \stackrel{\text { def }}{=}\left\{\eta \in L^{2}(\Omega): R_{p}(v, K) \geq R_{p}(u, K)+\langle\eta, v-u\rangle_{L^{2}(\Omega)}, \quad \forall v \in L^{2}(\Omega)\right\},
$$

and $\partial R_{p}(u, K)=\emptyset$ if $R_{p}(u, K)=+\infty$.

Lemma 3.1. Suppose that the assumptions of Theorem 3.1 hold. Then $u^{\star}$ is the unique solution to $\left(\mathcal{V} \mathcal{P}^{\lambda, p}\right)$ if and only if

$$
u^{\star}=\operatorname{prox}_{\lambda R_{p}(\cdot, K)}(g) \stackrel{\text { def }}{=}\left(\mathbf{I}+\lambda \partial R_{p}(\cdot, K)\right)^{-1}(g) .
$$


Moreover, the proximal mapping $\operatorname{prox}_{\lambda R_{p}(\cdot, K)}$ is non-expansive on $L^{2}(\Omega)$, i.e., for $g_{1}, g_{2} \in L^{2}(\Omega)$, the corresponding minimizers $u_{1}^{\star}, u_{2}^{\star} \in L^{2}(\Omega)$ obey

$$
\left\|u_{1}^{\star}-u_{2}^{\star}\right\|_{L^{2}(\Omega)} \leq\left\|g_{1}-g_{2}\right\|_{L^{2}(\Omega)} .
$$

A similar claim is easily obtained for $\left(\mathcal{V} \mathcal{P}_{n}^{\lambda, p}\right)$ as well.

Proof : The proof is again classical. By the first order optimality condition and since the squared $L^{2}(\Omega)$-norm is Fréchet differentiable, $u^{\star}$ is the unique solution to $\left(\mathcal{V} \mathcal{P}^{\lambda, p}\right)$ if, and only if the following monotone inclusion holds

$$
0 \in u^{\star}-g+\lambda \partial R_{p}\left(u^{\star}, K\right)
$$

The first claim then follows. Writing the subgradient inequality for $u_{1}^{\star}$ and $u_{2}^{\star}$ we have

$$
\begin{aligned}
& R_{p}\left(u_{2}^{\star}, K\right) \geq R_{p}\left(u_{1}^{\star}, K\right)+\frac{1}{\lambda}\left\langle g_{1}-u_{1}^{\star}, u_{2}^{\star}-u_{1}^{\star}\right\rangle_{L^{2}(\Omega)} \\
& R_{p}\left(u_{1}^{\star}, K\right) \geq R_{p}\left(u_{2}^{\star}, K\right)+\frac{1}{\lambda}\left\langle g_{2}-u_{2}^{\star}, u_{1}^{\star}-u_{2}^{\star}\right\rangle_{L^{2}(\Omega)}
\end{aligned}
$$

Adding these two inequalities we get

$$
\left\|u_{2}^{\star}-u_{1}^{\star}\right\|_{L^{2}(\Omega)}^{2} \leq\left\langle u_{2}^{\star}-u_{1}^{\star}, g_{2}-g_{1}\right\rangle_{L^{2}(\Omega)},
$$

and we conclude upon applying Cauchy-Schwartz inequality.

We now formally derive the directional derivative of $R_{p}(\cdot, K)$ when $\left.p \in\right] 1,+\infty[$. For this the symmetry assumption on $K$ is needed as well. Let $h \in L^{2}(\Omega)$. Then the following derivative exists

$$
\left.\frac{d}{d t} R_{p}(u+t h, K)\right|_{t=0}=\frac{1}{2} \int_{\Omega^{2}} K(x, y)|u(y)-u(x)|^{p-2}(u(y)-u(x))(v(y)-v(x)) d x d y .
$$

Since $K$ is symmetric, we apply the integration by parts formula in [30, Lemma A.1] (or split the integral in two terms and apply a change of variable $(x, y) \mapsto(y, x))$, to conclude that

$$
\left.\frac{d}{d t} R_{p}(u+t h, K)\right|_{t=0}=-\int_{\Omega^{2}} K(x, y)|u(y)-u(x)|^{p-2}(u(y)-u(x)) v(x) d x d y=\left\langle\boldsymbol{\Delta}_{p}^{K}, v\right\rangle_{L^{2}(\Omega)}
$$

where

$$
\boldsymbol{\Delta}_{p}^{K}=-\int_{\Omega^{2}} K(x, y)|u(y)-u(x)|^{p-2}(u(y)-u(x)) d y
$$

is precisely the nonlocal $p$-Laplacian operator, see $[1,30]$. This shows that under the above assumptions, $R_{p}(\cdot, K)$ is Fréchet differentiable (hence Gâteaux differentiable) on $L^{2}(\Omega)$ with Fréchet gradient $\boldsymbol{\Delta}_{p}^{K}$. 


\section{Error estimate for the discrete variational problem}

\subsection{Main result}

Our goal is to bound the difference between the unique minimizer of the continuum functional $E_{\lambda}(\cdot, g, K)$ defined on $L^{2}(\Omega)$ and the continuum extension by $I_{n}$ of that of $E_{n, \lambda}\left(\cdot, g_{n}, K_{n}\right)$. We are now ready to state the main result of this section.

Theorem 4.1. Suppose that $g \in L^{2}(\Omega)$ and $K$ is a nonnegative measurable, symmetric and bounded mapping. Let $u^{\star}$ and $u_{n}^{\star}$ be the unique minimizers of $\left(\mathcal{V} \mathcal{P}^{\lambda, p}\right)$ and $\left(\mathcal{V} \mathcal{P}_{n}^{\lambda, p}\right)$, respectively. Then, we have the following error bounds.

(i) If $p \in[1,2]$, then

$$
\begin{aligned}
\left\|I_{n} u_{n}^{\star}-u^{\star}\right\|_{L^{2}(\Omega)}^{2} & \leq C\left(\left\|g-I_{n} g_{n}\right\|_{L^{2}(\Omega)}^{2}+\left\|g-I_{n} g_{n}\right\|_{L^{2}(\Omega)}+\lambda\left\|K-I_{n} K_{n}\right\|_{L^{2}-p}\left(\Omega^{2}\right)\right. \\
& \left.+\lambda\left\|u^{\star}-I_{n} P_{n} u^{\star}\right\|_{L^{\frac{2}{3-p}}(\Omega)}\right)
\end{aligned}
$$

where $C$ is a positive constant independent of $n$ and $\lambda$.

(ii) If $\inf _{(x, y) \in \Omega^{2}} K(x, y) \geq \kappa>0$, then for any $p \in[1,+\infty[$,

$$
\begin{aligned}
\left\|I_{n} u_{n}^{\star}-u^{\star}\right\|_{L^{2}(\Omega)}^{2} & \leq C\left(\left\|g-I_{n} g_{n}\right\|_{L^{2}(\Omega)}^{2}+\left\|g-I_{n} g_{n}\right\|_{L^{2}(\Omega)}+\left\|K-I_{n} K_{n}\right\|_{L^{\infty}\left(\Omega^{2}\right)}\right. \\
& \left.+\lambda^{1 / p}\left\|u^{\star}-I_{n} P_{n} u^{\star}\right\|_{L^{p}(\Omega)}\right),
\end{aligned}
$$

where $C$ is a positive constant independent of $n$.

A few remarks are in order before proceeding to the proof.

\section{Remark 4.1.}

(i) Observe that $2 /(3-p) \leq p$ for $p \in[1,2]$. Thus by standard embeddings of $L^{q}(\Omega)$ spaces for $\Omega$ bounded, we have for $p \in[1,2]$

$$
\left\|K-I_{n} K_{n}\right\|_{L^{\frac{2}{2-p}}\left(\Omega^{2}\right)} \leq\left\|K-I_{n} K_{n}\right\|_{L^{\infty}\left(\Omega^{2}\right)} \text { and }\left\|u^{\star}-I_{n} P_{n} u^{\star}\right\|_{L^{\frac{2}{3-p}}(\Omega)} \leq\left\|u^{\star}-I_{n} P_{n} u^{\star}\right\|_{L^{p}(\Omega)},
$$

which means that our bound in (17) not only does not require an extra-assumption on $K$ but is also sharper than (18). The assumption on $K$ in the second statement seems difficult to remove or weaken. Whether this is possible or not is an open question that we leave to a future work.

(ii) We have made the dependence of the bound on $\lambda$ explicit on purpose. To see our motivation, assume that $g=u^{\dagger}+\varepsilon$, where $u^{\dagger} \in L^{2}(\Omega)$ is some true function and $\varepsilon \in L^{2}(\Omega)$ is some noise. Assume that $\partial R_{p}\left(u^{\dagger}, K\right) \neq \emptyset$, and let $\eta \in \partial R_{p}\left(u^{\dagger}, K\right)$, which is known in the inverse problem literature as a dual multiplier or certificate [52]. Then

$$
\left\|I_{n} u_{n}^{\star}-u^{\dagger}\right\|_{L^{2}(\Omega)} \leq\left\|I_{n} u_{n}^{\star}-u^{\star}\right\|_{L^{2}(\Omega)}+\left\|u^{\star}-u^{\dagger}\right\|_{L^{2}(\Omega)} .
$$


From [42, Proposition 3.41], one can show that

$$
\left\|u^{\star}-u^{\dagger}\right\|_{L^{2}(\Omega)} \leq 2\left(\|\varepsilon\|_{L^{2}(\Omega)}+\lambda\|\eta\|_{L^{2}(\Omega)}\right) .
$$

With the standard choice $\lambda \sim\|\varepsilon\|_{L^{2}(\Omega)}$ we have $\left\|u^{\star}-u^{\dagger}\right\|_{L^{2}(\Omega)}=O\left(\|\varepsilon\|_{L^{2}(\Omega)}\right)$, and thus $\left\|u^{\star}-u^{\dagger}\right\|_{L^{2}(\Omega)} \rightarrow 0$ as $\|\varepsilon\|_{L^{2}(\Omega)} \rightarrow 0$. Combining this with Theorem 4.1 and the fact that

$$
\left\|g-I_{n} g_{n}\right\|_{L^{2}(\Omega)} \leq\left\|u^{\dagger}-I_{n} P_{n} u^{\dagger}\right\|_{L^{2}(\Omega)}+2\|\varepsilon\|_{L^{2}(\Omega)},
$$

one obtains an error bound of $\left\|I_{n} u_{n}^{\star}-u^{\dagger}\right\|_{L^{2}(\Omega)}$ as function of $\|\varepsilon\|_{L^{2}(\Omega)}$ and the discretization error of $u^{\dagger}$ and $K$. This error bound is dominated by that of $u^{\dagger}$ and $K$ as $\|\varepsilon\|_{L^{2}(\Omega)} \rightarrow 0$ fast enough. Having said this, as our focus here is on numerical consistency, in the rest of the paper, the dependence of the error bound on $\lambda$ will be absorbed in the constants.

\section{PROOF :}

(i) Since $E_{\lambda}(\cdot, g, K)$ is a strongly convex function, we have

$$
\begin{aligned}
& \frac{1}{2 \lambda}\left\|I_{n} u_{n}^{\star}-u^{\star}\right\|_{L^{2}(\Omega)}^{2} \\
& \leq E_{\lambda}\left(I_{n} u_{n}^{\star}, g, K\right)-E_{\lambda}\left(u^{\star}, g, K\right) \\
& \leq\left(E_{\lambda}\left(I_{n} u_{n}^{\star}, g, K\right)-E_{n, \lambda}\left(u_{n}^{\star}, g_{n}, K_{n}\right)\right)-\left(E_{\lambda}\left(u^{\star}, g, K\right)-E_{n, \lambda}\left(u_{n}^{\star}, g_{n}, K_{n}\right)\right) .
\end{aligned}
$$

A closer inspection of $E_{\lambda}$ and $E_{n, \lambda}$ and equality (7) allows to assert that

$$
E_{\lambda}\left(I_{n} u_{n}^{\star}, I_{n} g_{n}, I_{n} K_{n}\right)=E_{n, \lambda}\left(u_{n}^{\star}, g_{n}, K_{n}\right) .
$$

Now, applying the Cauchy-Schwarz inequality and using (20), we have

$$
\begin{aligned}
E_{\lambda}\left(I_{n} u_{n}^{\star}, g, K\right) & =\frac{1}{2 \lambda}\left\|I_{n} u_{n}^{\star}-g\right\|_{L^{2}(\Omega)}^{2}+R_{p}\left(I_{n} u_{n}^{\star}, K\right) \\
& =\frac{1}{2 \lambda}\left\|I_{n} u_{n}^{\star}-I_{n} g_{n}\right\|_{L^{2}(\Omega)}^{2}+\frac{1}{\lambda}\left\langle I_{n} u_{n}^{\star}-I_{n} g_{n}, I_{n} g_{n}-g\right\rangle_{L^{2}(\Omega)} \\
& +\frac{1}{2 \lambda}\left\|I_{n} g_{n}-g\right\|_{L^{2}(\Omega)}^{2}+R_{p}\left(I_{n} u_{n}^{\star}, K\right) \\
& \leq \frac{1}{2 \lambda}\left\|I_{n} u_{n}^{\star}-I_{n} g_{n}\right\|_{L^{2}(\Omega)}^{2}+\frac{1}{\lambda}\left\|I_{n} u_{n}^{\star}-I_{n} g_{n}\right\|_{L^{2}(\Omega)}\left\|I_{n} g_{n}-g\right\|_{L^{2}(\Omega)} \\
& +\frac{1}{2 \lambda}\left\|I_{n} g_{n}-g\right\|_{L^{2}(\Omega)}^{2}+R_{p}\left(I_{n} u_{n}^{\star}, K\right) \\
& \leq E_{n, \lambda}\left(u_{n}^{\star}, g_{n}, K_{n}\right)+\frac{1}{2 \lambda}\left\|I_{n} g_{n}-g\right\|_{L^{2}(\Omega)}^{2} \\
& +\frac{1}{\lambda}\left\|I_{n} u_{n}^{\star}-I_{n} g_{n}\right\|_{L^{2}(\Omega)}\left\|I_{n} g_{n}-g\right\|_{L^{2}(\Omega)}+\left(R_{p}\left(I_{n} u_{n}^{\star}, K\right)-R_{p}\left(I_{n} u_{n}^{\star}, I_{n} K_{n}\right)\right) \\
& \leq E_{n, \lambda}\left(u_{n}^{\star}, g_{n}, K_{n}\right)+\frac{1}{2 \lambda}\left\|I_{n} g_{n}-g\right\|_{L^{2}(\Omega)}^{2} \\
& +\frac{1}{\lambda}\left\|I_{n} u_{n}^{\star}-I_{n} g_{n}\right\|_{L^{2}(\Omega)}\left\|I_{n} g_{n}-g\right\|_{L^{2}(\Omega)} \\
& +\frac{1}{2 p}\left|\int_{\Omega^{2}}\left(K(x, y)-I_{n} K_{n}(x, y)\right)\right| I_{n} u_{n}^{\star}(y)-\left.I_{n} u_{n}^{\star}(x)\right|^{p} d x d y \mid .
\end{aligned}
$$


As we suppose that $g \in L^{2}(\Omega)$ and since $u_{n}^{\star}$ is the (unique) minimizer of $E_{n, \lambda}\left(\cdot, g_{n}, K_{n}\right)$, it is immediate to see, using (20) and (8), that

$$
\begin{aligned}
\frac{1}{2 \lambda}\left\|I_{n} u_{n}^{\star}-I_{n} g_{n}\right\|_{L^{2}(\Omega)}^{2} & \leq E_{n, \lambda}\left(u_{n}^{\star}, g_{n}, K_{n}\right) \\
& \leq E_{n, \lambda}\left(0, g_{n}, K_{n}\right)=E_{\lambda}\left(0, I_{n} g_{n}, I_{n} K_{n}\right) \\
& =\frac{1}{2 \lambda}\left\|I_{n} g_{n}\right\|_{L^{2}(\Omega)}^{2}=\frac{1}{2 \lambda}\left\|I_{n} P_{n} g\right\|_{L^{2}(\Omega)}^{2} \leq \frac{1}{2 \lambda}\|g\|_{L^{2}(\Omega)}^{2}<+\infty
\end{aligned}
$$

and thus

$$
\left\|I_{n} u_{n}^{\star}-I_{n} g_{n}\right\|_{L^{2}(\Omega)} \leq\|g\|_{L^{2}(\Omega)} \stackrel{\text { def }}{=} C_{1}
$$

Since $p \in[1,2]$, by Hölder and triangle inequalities, and (13) applied to $I_{n} u_{n}^{\star}$, we have that

$$
\begin{aligned}
& \left|\int_{\Omega^{2}}\left(K(x, y)-I_{n} K_{n}(x, y)\right)\right| I_{n} u_{n}^{\star}(y)-\left.I_{n} u_{n}^{\star}(x)\right|^{p} d x d y \mid \\
& \leq\left\|K-I_{n} K_{n}\right\|_{L^{2-p}\left(\Omega^{2}\right)}\left(\int_{\Omega^{2}}\left|I_{n} u_{n}^{\star}(y)-I_{n} u_{n}^{\star}(x)\right|^{2} d x d y\right)^{p / 2} \\
& \leq 2^{p}\left\|I_{n} u_{n}^{\star}\right\|_{L^{2}(\Omega)}^{p}\left\|K-I_{n} K_{n}\right\|_{L^{\frac{2}{2-p}}\left(\Omega^{2}\right)} \\
& \leq 2^{2 p}\left\|I_{n} P_{n} g\right\|_{L^{2}(\Omega)}^{p}\left\|K-I_{n} K_{n}\right\|_{L^{\frac{2}{2-p}}\left(\Omega^{2}\right)}\left\|I_{L^{2-p}\left(\Omega^{2}\right)}=C_{2}\right\| K-I_{n} K_{n} \|_{L^{\frac{2}{2-p}}\left(\Omega^{2}\right)}, \\
& \leq 2^{2 p}\|g\|_{L^{2}(\Omega)}^{p}\left\|K-I_{n} K_{n}\right\|_{\frac{2}{2}}
\end{aligned}
$$

where $C_{2} \stackrel{\text { def }}{=} 2^{2 p} C_{1}^{p}$.

We now turn to bounding the second term on the right-hand side of (19). Using (8) and the fact that $u_{n}^{\star}$ is the (unique) minimizer of $\left(\mathcal{V} \mathcal{P}_{n}^{\lambda, p}\right)$, we have

$$
\begin{aligned}
E_{\lambda}\left(I_{n} u_{n}^{\star}, I_{n} g_{n}, I_{n} K_{n}\right) & \leq E_{\lambda}\left(I_{n} P_{n} u^{\star}, I_{n} g_{n}, I_{n} K_{n}\right) \\
& =\frac{1}{2 \lambda}\left\|I_{n} P_{n} u^{\star}-I_{n} P_{n} g\right\|_{L^{2}(\Omega)}^{2}+R_{p}\left(I_{n} P_{n} u^{\star}, I_{n} K_{n}\right) \\
& \leq \frac{1}{2 \lambda}\left\|u^{\star}-g\right\|_{L^{2}(\Omega)}^{2}+R_{p}\left(u^{\star}, K\right)+R_{p}\left(I_{n} P_{n} u^{\star}, I_{n} K_{n}\right)-R_{p}\left(u^{\star}, K\right) \\
& \leq E_{\lambda}\left(u^{\star}, g, K\right)+\left(R_{p}\left(I_{n} P_{n} u^{\star}, K\right)-R_{p}\left(u^{\star}, K\right)\right) \\
& +\left(R_{p}\left(I_{n} P_{n} u^{\star}, I_{n} K_{n}\right)-R_{p}\left(I_{n} P_{n} u^{\star}, K\right)\right) .
\end{aligned}
$$

We bound the second term on the right-hand side of (24) by applying the mean value theorem on $[a(x, y), b(x, y)]$ to the function $t \in \mathbb{R}^{+} \mapsto t^{p}$ with $a(x, y)=\left|u^{\star}(y)-u^{\star}(x)\right|$ and $b(x, y)=$ $\left|I_{n} P_{n} u^{\star}(y)-I_{n} P_{n} u^{\star}(x)\right|$. Let $\eta(x, y) \stackrel{\text { def }}{=} \rho a(x, y)+(1-\rho) b(x, y), \rho \in[0,1]$, be an intermediate 
value between $a(x, y)$ and $b(x, y)$. We then get

$$
\begin{aligned}
& \left|R_{p}\left(I_{n} P_{n} u^{\star}, K\right)-R_{p}\left(u^{\star}, K\right)\right| \\
& =\left|\int_{\Omega^{2}} K(x, y)\left(\left|I_{n} P_{n} u^{\star}(y)-I_{n} P_{n} u^{\star}(x)\right|^{p}-\left|u^{\star}(y)-u^{\star}(x)\right|^{p}\right) d x d y\right| \\
& =p\left|\int_{\Omega^{2}} K(x, y) \eta(x, y)^{p-1}\left(\left|I_{n} P_{n} u^{\star}(y)-I_{n} P_{n} u^{\star}(x)\right|-\left|u^{\star}(y)-u^{\star}(x)\right|\right) d x d y\right| \\
& \leq p C_{3} \int_{\Omega^{2}} \eta(x, y)^{p-1}\left|\left(I_{n} P_{n} u^{\star}(y)-u^{\star}(y)\right)-\left(I_{n} P_{n} u^{\star}(x)-u^{\star}(x)\right)\right| d x d y \\
& \leq 2 p C_{3} \int_{\Omega^{2}} \eta(x, y)^{p-1}\left|I_{n} P_{n} u^{\star}(x)-u^{\star}(x)\right| d x d y,
\end{aligned}
$$

where we used the triangle inequality, symmetry after the change of variable $(x, y) \mapsto(y, x)$, and boundedness of $K$, say $\|K\|_{L^{\infty}\left(\Omega^{2}\right)} \stackrel{\text { def }}{=} C_{3}$. Thus using Hölder and Jensen inequalities as well as (8), and arguing as in (23), leads to

$$
\begin{aligned}
& \left|R_{p}\left(I_{n} P_{n} u^{\star}, K\right)-R_{p}\left(u^{\star}, K\right)\right| \\
& \leq 2 p C_{3}\|\eta\|_{L^{2}\left(\Omega^{2}\right)}^{p-1}\left\|u^{\star}-I_{n} P_{n} u^{\star}\right\|_{L^{3-p}(\Omega)} \\
& \leq 2 p C_{3}\left(\rho\|a\|_{L^{2}\left(\Omega^{2}\right)}+(1-\rho)\|b\|_{L^{2}\left(\Omega^{2}\right)}\right)^{p-1}\left\|u^{\star}-I_{n} P_{n} u^{\star}\right\|_{L^{3-p}(\Omega)} \\
& \leq 2 p C_{3}\|a\|_{L^{2}\left(\Omega^{2}\right)}^{p-1}\left\|u^{\star}-I_{n} P_{n} u^{\star}\right\|_{L^{\frac{2}{3-p}}(\Omega)} \\
& \leq 2^{2 p-1} p C_{3}\|g\|_{L^{2}(\Omega)}^{p-1}\left\|u^{\star}-I_{n} P_{n} u^{\star}\right\|_{L^{\frac{2}{3-p}}(\Omega)}=C_{4}\left\|u^{\star}-I_{n} P_{n} u^{\star}\right\|_{L^{\frac{2}{3-p}}(\Omega)}
\end{aligned}
$$

where $C_{4} \stackrel{\text { def }}{=} 2^{2 p-1} p C_{1}^{p-1} C_{3}$.

To bound the last term on the right-hand side of (24), we follow the same steps as for establishing (23) and get

$$
\begin{aligned}
& \left|R_{p}\left(I_{n} P_{n} u^{\star}, I_{n} K_{n}\right)-R_{p}\left(I_{n} P_{n} u^{\star}, K\right)\right| \\
& \leq \int_{\Omega^{2}}\left|K(x, y)-I_{n} K_{n}(x, y)\right|\left|I_{n} P_{n} u^{\star}(y)-I_{n} P_{n} u^{\star}(x)\right|^{p} d x d y \\
& \leq C_{2}\left\|K-I_{n} K_{n}\right\|_{L^{\frac{2}{2-p}}\left(\Omega^{2}\right)}
\end{aligned}
$$

Finally, plugging (21), (22), (23), (24), (26) and (27) into (19), we get the desired result.

(ii) The case $p \geq 2$ follows the same proof steps, except that now, we need to modify inequalities (23), (26) and (27) which do not hold anymore.

Under our assumption on $K$, and using (14), (23) now reads

$$
\begin{aligned}
& \int_{\Omega^{2}}\left|K(x, y)-I_{n} K_{n}(x, y)\right|\left|I_{n} u_{n}^{\star}(y)-I_{n} u_{n}^{\star}(x)\right|^{p} d x d y \\
& \leq \kappa^{-1}\left\|K-I_{n} K_{n}\right\|_{L^{\infty}\left(\Omega^{2}\right)} \int_{\Omega^{2}} I_{n} K_{n}(x, y)\left|I_{n} u_{n}^{\star}(y)-I_{n} u_{n}^{\star}(x)\right|^{p} d x d y \\
& =\kappa^{-1}\left\|K-I_{n} K_{n}\right\|_{L^{\infty}\left(\Omega^{2}\right)} R_{p}\left(I_{n} u_{n}^{\star}, I_{n} K_{n}\right) \\
& \leq(2 \lambda \kappa)^{-1} C_{1}^{2}\left\|K-I_{n} K_{n}\right\|_{L^{\infty}\left(\Omega^{2}\right)},
\end{aligned}
$$


where $C_{1}=\|g\|_{L^{2}(\Omega)}$ as in the proof of (i).

We embark from the last line of (25) to which we apply Hölder inequality and then Jensen inequality as well as (8) to get

$$
\begin{aligned}
& \left|R_{p}\left(I_{n} P_{n} u^{\star}, K\right)-R_{p}\left(u^{\star}, K\right)\right| \\
& \leq 2 p C_{3}\|\eta\|_{L^{p}\left(\Omega^{2}\right)}^{(p-1)}\left\|u^{\star}-I_{n} P_{n} u^{\star}\right\|_{L^{p}(\Omega)} \\
& \leq 2 p C_{3}\|a\|_{L^{p}\left(\Omega^{2}\right)}^{(p-1)}\left\|u^{\star}-I_{n} P_{n} u^{\star}\right\|_{L^{p}(\Omega)} \\
& =2 p C_{3}\left(\int_{\Omega^{2}}\left|u^{\star}(y)-u^{\star}(x)\right|^{p} d x d y\right)^{(p-1) / p}\left\|u^{\star}-I_{n} P_{n} u^{\star}\right\|_{L^{p}(\Omega)} .
\end{aligned}
$$

Now, by assumption on $K$ and using again (14), we obtain

$$
\begin{aligned}
& \left|R_{p}\left(I_{n} P_{n} u^{\star}, K\right)-R_{p}\left(u^{\star}, K\right)\right| \\
& \leq 2 \kappa^{(1-p) / p} p C_{3}\left(\int_{\Omega^{2}} K(x, y)\left|u^{\star}(y)-u^{\star}(x)\right|^{p} d x d y\right)^{(p-1) / p}\left\|u^{\star}-I_{n} P_{n} u^{\star}\right\|_{L^{p}(\Omega)} \\
& =2 \kappa^{(1-p) / p} p C_{3}\left(R_{p}\left(u^{\star}, K\right)\right)^{(p-1) / p}\left\|u^{\star}-I_{n} P_{n} u^{\star}\right\|_{L^{p}(\Omega)} \\
& \leq 2(2 \lambda \kappa)^{(1-p) / p} p C_{3} C_{1}^{2(p-1) / p}\left\|u^{\star}-I_{n} P_{n} u^{\star}\right\|_{L^{p}(\Omega)}
\end{aligned}
$$

To get the new form of (27), we use (8), (14) and the assumption on $K$ to arrive at

$$
\begin{aligned}
& \left|R_{p}\left(I_{n} P_{n} u^{\star}, I_{n} K_{n}\right)-R_{p}\left(I_{n} P_{n} u^{\star}, K\right)\right| \\
& \leq \int_{\Omega^{2}}\left|K(x, y)-I_{n} K_{n}(x, y)\right|\left|I_{n} P_{n} u^{\star}(y)-I_{n} P_{n} u^{\star}(x)\right|^{p} d x d y \\
& \leq\left\|K-I_{n} K_{n}\right\|_{L^{\infty}\left(\Omega^{2}\right)} \int_{\Omega^{2}}\left|u^{\star}(y)-u^{\star}(x)\right|^{p} d x d y \\
& \leq \kappa^{-1}\left\|K-I_{n} K_{n}\right\|_{L^{\infty}\left(\Omega^{2}\right)} \int_{\Omega^{2}} K(x, y)\left|u^{\star}(y)-u^{\star}(x)\right|^{p} d x d y \\
& =\kappa^{-1}\left\|K-I_{n} K_{n}\right\|_{L^{\infty}\left(\Omega^{2}\right)} R_{p}\left(u^{\star}, K\right) \\
& \leq(2 \lambda \kappa)^{-1} C_{1}^{2}\left\|K-I_{n} K_{n}\right\|_{L^{\infty}\left(\Omega^{2}\right)}
\end{aligned}
$$

Plugging now (21), (22), (24), (28), (29) and (30) into (19), we conclude the proof.

\subsection{Regularity of the minimizer}

The error bound of Theorem 4.1 contain three terms: one which corresponds to the error in discretizing $g$, the second is the discretization error of the kernel $K$, and the last term reflects the discretization error of the minimizer $u^{\star}$ of the continuum problem $\left(\mathcal{V} \mathcal{P}^{\lambda, p}\right)$. Thus, this form is not convenient to transfer our bounds to networks on graph and establish convergence rates. Clearly, we need a control on the term $\left\|I_{n} P_{n} u^{\star}-u^{\star}\right\|_{L^{q}(\Omega)}$ on the right-hand side of (17)-(18). This is what 
we are about to do in the following key regularity lemma. In a nutshell, it states that if the kernel $K$ only depends on $|x-y|$ (as is the case for many kernels used in data processing), then as soon as the initial data $g$ belongs to some Lipschitz space, so does the minimizer $u^{\star}$.

Lemma 4.1. Suppose $g \in L^{\infty}(\Omega) \cap \operatorname{Lip}\left(s, L^{q}(\Omega)\right)$ with $\left.\left.s \in\right] 0,1\right]$ and $q \in[1,+\infty]$. Suppose furthermore that $K(x, y)=J(|x-y|)$, where $J$ is a nonnegative bounded measurable mapping on $\Omega$.

(i) If $q \in[1,2]$, then $u^{\star} \in \operatorname{Lip}\left(s q / 2, L^{q}(\Omega)\right)$.

(ii) If $q \in[2,+\infty]$, then $u^{\star} \in \operatorname{Lip}\left(s, L^{2}(\Omega)\right)$.

The boundedness assumption on $g$ can be removed for $q \geq 2$.

Proof : We denote the torus $\mathbb{T} \stackrel{\text { def }}{=} \mathbb{R} / 2 \mathbb{Z}$. For any function $u \in L^{2}(\Omega)$, we denote by $\bar{u} \in L^{2}(\mathbb{T})$ its periodic extension such that

$$
\bar{u}(x)= \begin{cases}u(x) \quad \text { if } & x \in[0,1] \\ u(2-x) & \text { if } \quad x \in] 1,2]\end{cases}
$$

In the rest of the proof, we use letters with bars to indicate functions defined on $\mathbb{T}$.

Let us define

$$
\bar{E}_{\lambda / 2}(\bar{v}, \bar{g}, \bar{J}) \stackrel{\text { def }}{=} \frac{1}{\lambda}\|\bar{v}-\bar{g}\|_{L^{2}(\mathbb{T})}^{2}+\bar{R}_{p}(\bar{v}, \bar{J})
$$

where

$$
\bar{R}_{p}(\bar{v}, \bar{J}) \stackrel{\text { def }}{=} \frac{1}{2 p} \int_{\mathbb{T}^{2}} \bar{J}(|x-y|)|\bar{v}(y)-\bar{v}(x)|^{p} d x d y .
$$

Consider the following minimization problem

$$
\min _{\bar{v} \in L^{2}(\mathbb{T})} \bar{E}_{\lambda / 2}(\bar{v}, \bar{g}, \bar{J})
$$

which also has a unique minimizer by arguments similar to those of Theorem 3.1. Since $u^{\star}$ is the unique minimizer of $\left(\mathcal{V} \mathcal{P}^{\lambda, p}\right)$, we have, using (31),

$$
\begin{aligned}
\bar{E}_{\lambda / 2}\left(\overline{u^{\star}}, \bar{g}, \bar{J}\right) & =\frac{2}{\lambda}\left\|u^{\star}-g\right\|_{L^{2}(\Omega)}^{2}+4 R_{p}\left(u^{\star}, J\right) \\
& =4 E_{\lambda}\left(u^{\star}, g, J\right) \\
& <4 E_{\lambda}(v, g, J), \forall v \neq u^{\star} \\
& =\bar{E}_{\lambda / 2}(\bar{v}, \bar{g}, \bar{J}), \forall \bar{v} \neq \overline{u^{\star}}
\end{aligned}
$$

which shows that $\overline{u^{\star}}$ is the unique minimizer of (32). Then, we have via Lemma 3.1

$$
\overline{u^{\star}}=\operatorname{prox}_{\lambda / 2} \bar{R}_{p}(\cdot, \bar{J})(\bar{g})
$$

We define the translation operator

$$
\left(T_{h} v\right)(x)=v(x+h), \forall h \in \mathbb{R} .
$$


Now, using our assumption on the kernel $K$, that is $K(x, y)=J(|x-y|)$ (then invariant by translation), and periodicity of the functions on $\mathbb{T}$, we have

$$
\begin{aligned}
\bar{E}_{\lambda / 2}\left(\bar{v}, T_{h} \bar{g}, \bar{J}\right) & =\frac{1}{\lambda}\left\|\bar{v}-T_{h} \bar{g}\right\|_{L^{2}(\mathbb{T})}^{2}+\bar{R}_{p}(\bar{v}, \bar{J}) \\
& =\frac{1}{\lambda}\left\|T_{h}\left(T_{-h} \bar{v}-\bar{g}\right)\right\|_{L^{2}(\mathbb{T})}^{2} \\
& +\int_{\mathbb{T}^{2}} \bar{J}(|x-y|)|\bar{v}((y+h)-h)-\bar{v}((x+h)-h)|^{p} d x d y \\
& =\frac{1}{\lambda}\left\|T_{-h} \bar{v}-\bar{g}\right\|_{L^{2}(\mathbb{T})}^{2}+\int_{\mathbb{T}^{2}} \bar{J}(|x-y|)\left|T_{-h} \bar{v}(y)-T_{-h} \bar{v}(x)\right|^{p} d x d y \\
& =\bar{E}_{\lambda / 2}\left(T_{-h} \bar{v}, \bar{g}, \bar{J}\right) .
\end{aligned}
$$

This implies that the unique minimizer $\bar{v}^{\star}$ of $\bar{E}_{\lambda / 2}\left(\cdot, T_{h} \bar{g}, \bar{J}\right)$ given by (see Lemma 3.1)

$$
\bar{v}^{\star}=\operatorname{prox}_{\lambda / 2 \bar{R}_{p}(\cdot, \bar{J})}\left(T_{h} \bar{g}\right),
$$

is also the unique minimizer of $\bar{E}_{\lambda / 2}\left(T_{-h}, \bar{g}, \bar{J}\right)$. But since $\bar{E}_{\lambda / 2}(\cdot, \bar{g}, \bar{J})$ has a unique minimizer $\overline{u^{\star}}$, we deduce from (34) and (35) that

$$
T_{h} \operatorname{prox}_{\lambda / 2 \bar{R}_{p}(\cdot, \bar{J})}(\bar{g})=\operatorname{prox}_{\lambda / 2 \bar{R}_{p}(\cdot, \bar{J})}\left(T_{h} \bar{g}\right) .
$$

That is, the proximal mapping of $\lambda / 2 \bar{R}_{p}(\cdot, \bar{J})$ commutes with translation.

We now split the two cases of $q$.

(i) For $q \in[1,2]$ : combining (34), (36), (16), [30, Lemma C.1] and that $L^{2}(\Omega) \subset L^{q}(\Omega)$, we have

$$
\begin{aligned}
\left\|T_{h} \overline{u^{\star}}-\overline{u^{\star}}\right\|_{L^{q}(\mathbb{T})} & =\left\|\operatorname{prox}_{\lambda / 2 \bar{R}_{p}(\cdot, \bar{J})}\left(T_{h} \bar{g}\right)-\operatorname{prox}_{\lambda / 2 \bar{R}_{p}(\cdot, \bar{J})}(\bar{g})\right\|_{L^{q}(\mathbb{T})} \\
& \leq 2^{1 / q-1 / 2}\left\|\operatorname{prox}_{\lambda / 2 \bar{R}_{p}(\cdot, \bar{J})}\left(T_{h} \bar{g}\right)-\operatorname{prox}_{\lambda / 2 \bar{R}_{p}(\cdot, \bar{J})}(\bar{g})\right\|_{L^{2}(\mathbb{T})} \\
& \leq 2^{1 / q-1 / 2}\left\|T_{h} \bar{g}-\bar{g}\right\|_{L^{2}(\mathbb{T})} \\
& \leq 2^{1 / q-1 / 2}\left(2\|g\|_{L^{\infty}(\Omega)}\right)^{1-q / 2}\left\|T_{h} \bar{g}-\bar{g}\right\|_{L^{q}(\mathbb{T})}^{q / 2} \\
& =2^{1 / 2}\left(\|g\|_{L^{\infty}(\Omega)}\right)^{1-q / 2}=C_{1}\left\|T_{h} \bar{g}-\bar{g}\right\|_{L^{q}(\mathbb{T})^{q}}^{q / 2}
\end{aligned}
$$

Let $\Omega_{h} \stackrel{\text { def }}{=}\{x \in \Omega: x+h \in \Omega\}$. Recalling the modulus of smoothness in (10), we have

$$
\begin{aligned}
w\left(u^{\star}, t\right)_{q} \stackrel{\text { def }}{=} \sup _{|h|<t}\left\|T_{h} u^{\star}-u^{\star}\right\|_{L^{q}\left(\Omega_{h}\right)} & \leq C_{2} \sup _{|h|<t}\left\|T_{h} \overline{u^{\star}}-\overline{u^{\star}}\right\|_{L^{q}(\mathbb{T})} \\
& \leq C_{1} C_{2}\left(\sup _{|h|<t}\left\|T_{h} \bar{g}-\bar{g}\right\|_{L^{q}(\mathbb{T})}\right)^{q / 2} \\
& =C_{1} C_{2} w(\bar{g}, t)_{q}^{q / 2} \\
& \leq C_{1} C_{2}\left(C_{3} w(g, t)_{q}\right)^{q / 2}
\end{aligned}
$$

We get the last inequality by applying the Whitney extension theorem $[18$, Ch. 6 , Theorem 4.1]. Invoking Definition 2.2, there exists a constant $C>0$ such that

$$
\left|u^{\star}\right|_{\operatorname{Lip}\left(s q / 2, L^{q}(\Omega)\right)} \stackrel{\text { def }}{=} \sup _{t>0} t^{-s q / 2} w\left(u^{\star}, t\right)_{q} \leq C\left(\sup _{t>0} t^{-s} w(g, t)_{q}\right)^{q / 2} \leq C|g|_{\operatorname{Lip}\left(s, L^{q}(\Omega)\right)}^{q / 2},
$$


whence the claim follows after observing that $u^{\star} \in L^{2}(\Omega) \subset L^{q}(\Omega)$.

(ii) For $q \in[2,+\infty]$, we combine (34), (36), (16), and that now $L^{q}(\Omega) \subset L^{2}(\Omega)$, to get

$$
\left\|T_{h} \overline{u^{\star}}-\overline{u^{\star}}\right\|_{L^{2}(\mathbb{T})} \leq\left\|T_{h} \bar{g}-\bar{g}\right\|_{L^{2}(\mathbb{T})} \leq 2^{1 / 2-1 / q}\left\|T_{h} \bar{g}-\bar{g}\right\|_{L^{q}(\mathbb{T})} .
$$

The rest of the proof is similar to that of (i).

In view of the regularity Lemma 4.1 and Theorem 4.1, one can derive convergence rates but only for $p \in[1,2]$. Indeed, the approximation bounds of Lemma 2.2 cannot be applied to $u^{\star}-I_{n} P_{n} u^{\star}$ for $p \geq 2$ since the bound in Theorem 4.1(ii) is in the $L^{p}(\Omega)$ norm while Lemma 4.1 proves that $u^{\star}$ is only in $\operatorname{Lip}\left(s q / 2, L^{2}(\Omega)\right)$. In particular, one cannot invoke (12) since there is no guarantee that $u^{\star}$ is bounded. This is the reason why in the rest of the paper, we will only focus on the case $p \in[1,2]$.

\section{Application to dense deterministic graph sequences}

The graph models we will consider here were used first in [37] and then [30] to study networks on graphs for the evolution Cauchy problem, governed by the $p$-Laplacian in [30]. Throughout the section, we suppose that $p \in[1,2]$.

\subsection{Networks on simple graphs}

We first consider the case of a sequence of simple graphs converging to a $\{0,1\}$ graphon. Briefly speaking, we define a sequence of simple graphs $G_{n}=\left(V\left(G_{n}\right), E\left(G_{n}\right)\right)$ such that $V\left(G_{n}\right)=[n]$ and

$$
E\left(G_{n}\right)=\left\{(i, j) \in[n]^{2}: \Omega_{i j}^{(n)} \cap \overline{\operatorname{supp}(K)} \neq \emptyset\right\},
$$

where $\overline{\operatorname{supp}(K)}$ is the closure of the support of $K$

$$
\operatorname{supp}(K)=\left\{(x, y) \in \Omega^{2}: K(x, y) \neq 0\right\} .
$$

As we have mentioned in Section 2.2, the kernel $K$ represents the corresponding graph limit, that is the limit as $n \rightarrow \infty$ of the function $K_{G_{n}}: \Omega^{2} \rightarrow\{0,1\}$ such that

$$
K_{G_{n}}(x, y)= \begin{cases}1, & \text { if }(i, j) \in E\left(G_{n}\right) \quad \text { and } \quad(x, y) \in \Omega_{i j}^{(n)}, \\ 0 & \text { otherwise }\end{cases}
$$

As $n \rightarrow \infty,\left\{K_{G_{n}}\right\}_{n \in \mathbb{N}^{*}}$ converges to the $\{0,1\}$-valued mapping $K$ whose support is defined by (40). With this construction, the discrete counterpart of $\left(\mathcal{V} \mathcal{P}^{\lambda, p}\right)$ on the graph $G_{n}$ is then given by

$$
\min _{u_{n} \in \mathbb{R}^{n}}\left\{E_{n, \lambda}\left(u_{n}, g_{n}, K_{n}\right) \stackrel{\text { def }}{=} \frac{1}{2 \lambda n}\left\|u_{n}-g_{n}\right\|_{2}^{2}+\frac{1}{2 p n^{2}} \sum_{(i, j) \in E\left(G_{n}\right)}\left|u_{n j}-u_{n i}\right|^{p}\right\}, \quad\left(\mathcal{V} \mathcal{P}_{s, n}^{\lambda, p}\right)
$$

where the initial data $g_{n}$ is given by (4). 
For this model, $I_{n} K_{n}(x, y)$ is the piecewise constant function such that for $(x, y) \in \Omega_{i j}^{(n)},(i, j) \in$ $[n]^{2}$

$$
I_{n} K_{n}(x, y)=\left\{\begin{array}{l}
\frac{1}{\left|\Omega_{i j}^{(n)}\right|} \int_{\Omega_{i j}^{(n)}} K(x, y) d x d y \quad \text { if } \quad \Omega_{i j}^{(n)} \cap \overline{\operatorname{supp}(K)} \neq \emptyset \\
0 \quad \text { otherwise. }
\end{array}\right.
$$

Relying on what we did in [30], the rate of convergence of the solution of the discrete problem to the solution of the limiting problem depends on the regularity of the boundary $\operatorname{bd}(\overline{\operatorname{supp}(K)})$ of the closure of the support. Following [37], we recall the upper box-counting (or Minkowski-Bouligand) dimension of bd( $\overline{\operatorname{supp}(K)})$ as a subset of $\mathbb{R}^{2}$ :

$$
\rho \stackrel{\text { def }}{=} \operatorname{dim}_{B}(\operatorname{bd}(\overline{\operatorname{supp}(K)}))=\limsup _{\delta \rightarrow 0} \frac{\log N_{\delta}(\operatorname{bd}(\overline{\operatorname{supp}(K)}))}{-\log \delta},
$$

where $N_{\delta}(\mathrm{bd}(\overline{\operatorname{supp}(K)}))$ is the number of cells of a $(\delta \times \delta)$-mesh that intersect bd( $\left.\overline{\operatorname{supp}(K)}\right)$ (see $[24])$.

Theorem 5.1. Assume that $p \in[1,2], g \in L^{2}(\Omega)$. Let $u^{\star}$ and $u_{n}^{\star}$ be the unique minimizers of $\left(\mathcal{V} \mathcal{P}^{\lambda, p}\right)$ and $\left(\mathcal{V} \mathcal{P}_{s, n}^{\lambda, p}\right)$, respectively. Then, the following hold.

(i) We have

$$
\left\|I_{n} u_{n}^{\star}-u^{\star}\right\|_{L^{2}(\Omega)} \underset{n \rightarrow+\infty}{\longrightarrow} 0
$$

(ii) For $p \in\left[1,2\left[\right.\right.$ : assume moreover $g \in L^{\infty}(\Omega) \cap \operatorname{Lip}\left(s, L^{q}(\Omega)\right)$, with $\left.\left.s \in\right] 0,1\right]$ and $q \in[2 /(3-p), 2]$, that $\rho \in\left[0,2\left[\right.\right.$ and that $K(x, y)=J(|x-y|), \forall(x, y) \in \Omega^{2}$, with $J$ a nonnegative bounded measurable mapping on $\Omega$. Then for any $\epsilon>0$ there exists $N(\epsilon) \in \mathbb{N}$ such that for any $n \geq N(\epsilon)$

$$
\left\|I_{n} u_{n}^{\star}-u^{\star}\right\|_{L^{2}(\Omega)}^{2} \leq C n^{-\min \left(s q / 2,(2-p)\left(1-\frac{\rho+\epsilon}{2}\right)\right)},
$$

where $C$ is a positive constant independent of $n$.

\section{Proof :}

(i) In view of (4), by the Lebesgue differentiation theorem (see e.g. [39, Theorem 3.4.4]), we have

$$
I_{n} g_{n}(x) \underset{n \rightarrow \infty}{\longrightarrow} g(x), \quad I_{n} P_{n} u^{\star}(x) \underset{n \rightarrow \infty}{\longrightarrow} u^{\star}(x) \text { and } I_{n} K_{n}(x, y) \underset{n \rightarrow \infty}{\longrightarrow} K(x, y)
$$

almost everywhere on $\Omega$ and $\Omega^{2}$, respectively. Combining this with Fatou's lemma and (8), we have

$$
\begin{aligned}
\|g\|_{L^{2}(\Omega)}^{2}=\int_{\Omega}\left|\lim _{n \rightarrow \infty} I_{n} g_{n}(x)\right|^{2} d x & =\int_{\Omega} \liminf _{n \rightarrow \infty}\left|I_{n} g_{n}(x)\right|^{2} d x \\
& \leq \liminf _{n \rightarrow \infty}\left\|I_{n} g_{n}\right\|_{L^{2}(\Omega)}^{2} \\
& \leq \limsup _{n \rightarrow \infty}\left\|I_{n} P_{n} g\right\|_{L^{2}(\Omega)}^{2} \leq\|g\|_{L^{2}(\Omega)}^{2}
\end{aligned}
$$


which entails that $\lim _{n \rightarrow \infty}\left\|I_{n} g_{n}\right\|_{L^{2}(\Omega)}=\|g\|_{L^{2}(\Omega)}$. Similarly, we have

$$
\lim _{n \rightarrow \infty}\left\|I_{n} P_{n} u^{\star}\right\|_{L^{\frac{2}{3-p}}(\Omega)}=\left\|u^{\star}\right\|_{L^{\frac{2}{3-p}}(\Omega)} .
$$

Since $g \in L^{2}(\Omega), u^{\star} \in L^{2}(\Omega) \subset L^{\frac{2}{3-p}}(\Omega)$ (Theorem 3.1), we are in position to apply the Riesz-Scheffé lemma [33, Lemma 2] to deduce that

$$
\left\|I_{n} g_{n}-g\right\|_{L^{2}(\Omega)} \underset{n \rightarrow \infty}{\longrightarrow} 0 \text { and }\left\|I_{n} P_{n} u^{\star}-u^{\star}\right\|_{L^{\frac{2}{3-p}}(\Omega)} \underset{n \rightarrow \infty}{\longrightarrow} 0 .
$$

Observe that for simple graphs, $I_{n} K_{n}$ is not an orthogonal projection of $K$ (see (41)) and thus, the above argument proof used for $g$ and $u^{\star}$ does not hold. We argue however using the fact that $K$ is bounded, $|\Omega|<\infty$, and that $\forall n$ and $(x, y) \in \Omega^{2},\left|I_{n} K_{n}(x, y)\right| \leq\|K\|_{L^{\infty}(\Omega)}$. We can thus invoke the dominated convergence theorem to get that

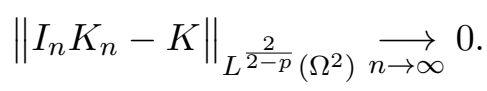

Passing to the limit in (17), we get the claim.

(ii) In the following $C$ is any positive constant independent of $n$. Since $g \in L^{\infty}(\Omega) \cap \operatorname{Lip}\left(s, L^{q}(\Omega)\right)$, $q \leq 2$, and we are dealing with a uniform partition of $\Omega\left(\left|\Omega_{i}^{(n)}\right|=1 / n, \forall i \in[n]\right)$, we get using inequality (12) that

$$
\left\|I_{n} g_{n}-g\right\|_{L^{2}(\Omega)} \leq C n^{-s \min (1, q / 2)}=C n^{-s q / 2} .
$$

By Lemma 4.1(i), we have $u^{\star} \in \operatorname{Lip}\left(s q / 2, L^{q}(\Omega)\right)$, and it follows from (11) and the fact that $q \geq 2 /(3-p)$ that

$$
\left\|I_{n} P_{n} u^{\star}-u^{\star}\right\|_{L^{3-p}(\Omega)} \leq\left\|I_{n} P_{n} u^{\star}-u^{\star}\right\|_{L^{q}(\Omega)} \leq C n^{-s q / 2} .
$$

Combining (43) and (44), we get

$$
\begin{aligned}
\left\|I_{n} g_{n}-g\right\|_{L^{2}(\Omega)}^{2}+\left\|I_{n} g_{n}-g\right\|_{L^{2}(\Omega)}+\left\|I_{n} P_{n} u^{\star}-u^{\star}\right\|_{L^{\frac{2}{3-p}}(\Omega)} & \leq C\left(n^{-s q}+n^{-s q / 2}\right) \\
& \leq C n^{-s q / 2} .
\end{aligned}
$$

It remains to bound $\left\|K-I_{n} K_{n}\right\|_{L^{\frac{2}{2-p}}\left(\Omega^{2}\right)}$. For that, consider the set of discrete cells $\Omega_{i j}^{(n)}$ overlying the boundary of the support of $K$

$$
S(n)=\left\{(i, j) \in[n]^{2}: \Omega_{i j}^{(n)} \cap \operatorname{bd}(\overline{\operatorname{supp}(K)}) \neq \emptyset\right\} \text { and } C(n)=|S(n)| .
$$

For any $\epsilon>0$ and sufficiently large $n$, we have

$$
C(n) \leq n^{\rho+\epsilon} .
$$

It is easy to see that $K$ and $I_{n} K_{n}$ coincide almost everywhere on cells $\Omega_{i j}^{(n)}$ such that $(i, j) \notin$ $S(n)$. Thus, for any $\epsilon>0$ and all sufficiently large $n$, we have

$$
\left\|K-I_{n} K_{n}\right\|_{L^{\frac{2}{2-p}}\left(\Omega^{2}\right)}^{\frac{2}{2-p}} \leq C(n) n^{-2} \leq n^{-2\left(1-\frac{\rho+\epsilon}{2}\right)} .
$$

Inserting (45) and (46) into (17), the desired result follows. 


\subsection{Networks on weighted graphs}

We now turn to the more general class of deterministic weighted graph sequences. The kernel $K$ is used to assign weights to the edges of the graphs considered below, we allow only positive weights. These weights $K_{n i j}$ are obtained by averaging $K$ over the cells in the partition $\mathcal{Q}_{n}$ following (4), and $I_{n} K_{n}$ is given by (5).

Proceeding similarly to the proof of statement (i) of Theorem 5.1, we conclude immediately that

$$
\left\|I_{n} u_{n}^{\star}-u\right\|_{L^{2}(\Omega)} \underset{n \rightarrow+\infty}{\longrightarrow} 0 .
$$

We are rather interested now in quantifying the rate of convergence in (17). To do so, we need to add some regularity assumptions on the kernel $K$.

Theorem 5.2. Let $p \in\left[1,2\left[\right.\right.$, and assume that $g \in L^{\infty}(\Omega) \cap \operatorname{Lip}\left(s, L^{q}(\Omega)\right)$, with $\left.\left.s \in\right] 0,1\right]$ and $q \in[2 /(3-p), 2]$. Suppose moreover that $K(x, y)=J(|x-y|), \forall(x, y) \in \Omega^{2}$, with $J$ a nonnegative bounded measurable mapping on $\Omega$. Let $u^{\star}$ and $u_{n}^{\star}$ be the unique minimizers of $\left(\mathcal{V P}{ }^{\lambda, p}\right)$ and $\left(\mathcal{V P}{ }_{n}^{\lambda, p}\right)$, respectively. Then, the following error bounds hold.

(i) If $p \in\left[1,2\left[, K \in \operatorname{Lip}\left(s^{\prime}, L^{q^{\prime}}\left(\Omega^{2}\right)\right)\right.\right.$ and $\left.\left.\left(s^{\prime}, q^{\prime}\right) \in\right] 0,1\right] \times[1,+\infty[$, then

$$
\left\|I_{n} u_{n}^{\star}-u^{\star}\right\|_{L^{2}(\Omega)}^{2} \leq C n^{-\min \left(s q / 2, s^{\prime}, s^{\prime} q^{\prime}(1-p / 2)\right)} .
$$

where $C$ is a positive constant independent of $n$.

In particular, if $g \in L^{\infty}(\Omega) \cap \mathrm{BV}(\Omega)$ and $K \in L^{\infty}\left(\Omega^{2}\right) \cap \mathrm{BV}\left(\Omega^{2}\right)$, then

$$
\left\|I_{n} u_{n}^{\star}-u\right\|_{L^{2}(\Omega)}^{2}=O\left(n^{p / 2-1}\right) .
$$

(ii) If $p \in[1,2], K \in \operatorname{Lip}\left(s^{\prime}, L^{q^{\prime}}\left(\Omega^{2}\right)\right)$ and $\left.\left.\left(s^{\prime}, q^{\prime}\right) \in\right] 0,1\right] \times[2 /(2-p),+\infty]$, then

$$
\left\|I_{n} u_{n}^{\star}-u^{\star}\right\|_{L^{2}(\Omega)}^{2} \leq C n^{-\min \left(s q / 2, s^{\prime}\right)} .
$$

where $C$ is a positive constant independent of $n$.

In particular, if $g \in L^{\infty}(\Omega) \cap \mathrm{BV}(\Omega)$ then

$$
\left\|I_{n} u_{n}^{\star}-u\right\|_{L^{2}(\Omega)}^{2}=O\left(n^{-\min \left(1 / 2, s^{\prime}\right)}\right) .
$$

Proof : In the following $C$ is any positive constant independent of $n$. Under the setting of the theorem, for all cases, (45) still holds. It remains to bound $\left\|K-I_{n} K_{n}\right\|_{L^{\frac{2}{2-p}}\left(\Omega^{2}\right)}$. This is achieved using (12) for case (i) and (11) for case (ii), which yields

$$
\begin{cases}\left\|K-I_{n} K_{n}\right\|_{L^{\frac{2}{2-p}}\left(\Omega^{2}\right)} \leq C n^{-s^{\prime} \min \left(1, q^{\prime}(1-p / 2)\right)} \quad \text { for case (i), } \\ \left\|K-I_{n} K_{n}\right\|_{L^{\frac{2}{2-p}}\left(\Omega^{2}\right)} \leq\left\|K-I_{n} K_{n}\right\|_{L^{q^{\prime}}\left(\Omega^{2}\right)} \leq C n^{-s^{\prime}} & \text { for case (ii). }\end{cases}
$$

Plugging (45) and (51) into (17), the bounds (47) and (49) follow. 
We know that $\operatorname{BV}(\Omega) \subset \operatorname{Lip}\left(1 / 2, L^{2}(\Omega)\right)$. Thus setting $s=s^{\prime}=1 / 2$ and $q=q^{\prime}=2$ in (47), and observing that $1-p / 2 \in[0,1 / 2]$, the bound (48) follows. That of (50) is immediate.

When $p=1$ (i.e., nonlocal total variation), $g \in L^{\infty}(\Omega) \cap \operatorname{Lip}\left(s, L^{2}(\Omega)\right)$ and $K$ is a sufficiently smooth function, one can infer from Theorem 5.2 that the solution to the discrete problem $\left(\mathcal{V P}{ }_{n}^{\lambda, p}\right)$ converges to that of the continuum problem $\left(\mathcal{V} \mathcal{P}^{\lambda, p}\right)$ at the rate $O\left(n^{-s}\right)$. Moreover, if $g \in L^{\infty}(\Omega) \cap$ $\mathrm{BV}(\Omega)$, then the best convergence rate is $O\left(n^{-1 / 2}\right)$ which is attained precisely for $p=1$.

\section{Application to random inhomogeneous graph sequences}

We now turn to applying our bounds of Theorem 5.2 to networks on random inhomogeneous graphs.

We start with the description of the random graph model we will use. This random graph model is motivated by the construction of inhomogeneous random graphs in [5, 8]. It is generated as follows.

Definition 6.1. Fix $n \in \mathbb{N}^{*}$ and let $K$ be a symmetric measurable function on $\Omega^{2}$. Generate the graph $G_{n}=\left(V\left(G_{n}\right), E\left(G_{n}\right)\right) \stackrel{\text { def }}{=} G_{q_{n}}(n, K)$ as follows:

1) Generate $n$ independent and identically distributed (i.i.d.) random variables $\mathbf{X} \stackrel{\text { def }}{=}\left(\mathbf{X}_{1}, \cdots, \mathbf{X}_{n}\right)$ from the uniform distribution on $\Omega$. Let $\left\{\mathbf{X}_{(i)}\right\}_{i=1}^{n}$ be the order statistics of the random vector $\mathbf{X}$, i.e. $\mathbf{X}_{(i)}$ is the $i$-th smallest value.

2) Conditionally on $\mathbf{X}$, join each pair $(i, j) \in[n]^{2}$ of vertices independently, with probability $q_{n} \hat{K}_{n i j}^{\mathbf{X}}$, i.e. for every $(i, j) \in[n]^{2}, i \neq j$,

$$
\mathbb{P}\left((i, j) \in E\left(G_{n}\right) \mid \mathbf{X}\right)=q_{n} \stackrel{\wedge}{K}_{n i j}^{\mathbf{X}}
$$

where

$$
\stackrel{\wedge}{K}_{n i j}^{\mathbf{X}} \stackrel{\text { def }}{=} \min \left(\frac{1}{\left|\Omega_{n i j}^{\mathbf{X}}\right|} \int_{\Omega_{n i j}^{\mathbf{X}}} K(x, y) d x d y, 1 / q_{n}\right)
$$

and

$$
\left.\left.\left.\left.\Omega_{n i j}^{\mathbf{X}} \stackrel{\text { def }}{=}\right] \mathbf{X}_{(i-1)}, \mathbf{X}_{(i)}\right] \times\right] \mathbf{X}_{(j-1)}, \mathbf{X}_{(j)}\right]
$$

where $q_{n}$ is nonnegative and uniformly bounded in $n$.

A graph $G_{q_{n}}(n, K)$ generated according to this procedure is called a $K$-random inhomogeneous graph generated by a random sequence $\mathbf{X}$.

We denote by $\mathbf{x}=\left(\mathbf{x}_{1}, \cdots, \mathbf{x}_{n}\right)$ the realization of $\mathbf{X}$. To lighten the notation, we also denote

$$
\left.\left.\left.\left.\left.\left.\left.\left.\Omega_{n i}^{\mathbf{X}} \stackrel{\text { def }}{=}\right] \mathbf{X}_{(i-1)}, \mathbf{X}_{(i)}\right], \quad \Omega_{n i}^{\mathbf{x}} \stackrel{\text { def }}{=}\right] \mathbf{x}_{(i-1)}, \mathbf{x}_{(i)}\right], \quad \text { and } \Omega_{n i j}^{\mathbf{x}} \stackrel{\text { def }}{=}\right] \mathbf{x}_{(i-1)}, \mathbf{x}_{(i)}\right] \times\right] \mathbf{x}_{(j-1)}, \mathbf{x}_{(j)}\right] \quad i, j \in[n] .
$$

As the realization of the random vector $\mathbf{X}$ is fixed, we define

$$
\stackrel{\wedge}{K}_{n i j}^{\mathbf{x}} \stackrel{\text { def }}{=} \min \left(\frac{1}{\left|\Omega_{n i j}^{\mathbf{x}}\right|} \int_{\Omega_{n i j}^{\mathbf{x}}} K(x, y) d x d y, 1 / q_{n}\right), \quad \forall(i, j) \in[n]^{2}, \quad i \neq j .
$$


In the rest of the paper, the following random variables will be useful. Let $\Lambda_{n}=\left\{\Lambda_{n i j}\right\}_{(i, j) \in[n]^{2}, i \neq j}$ be a collection of independent random variables such that $q_{n} \Lambda_{n i j}$ follows a Bernoulli distribution with parameter $q_{n} \hat{K}_{n i j}^{\mathbf{x}}$. We consider the independent random variables $\Upsilon_{i j}$ such that the distribution of $q_{n} \Upsilon_{i j}$ conditionally on $\mathbf{X}=\mathbf{x}$ is that of $q_{n} \Lambda_{n i j}$. Thus $q_{n} \Upsilon_{i j}$ follows

a Bernoulli distribution with parameter $\mathbb{E}\left(q_{n} \stackrel{\wedge}{K}_{n i j}^{\mathbf{X}}\right)$, where $\mathbb{E}(\cdot)$ is the expectation operator (here with respect to the distribution of $\mathbf{X}$ ).

We put the following assumptions on the parameters of the graph sequence $\left\{G_{q_{n}}(n, K)\right\}_{n \in \mathbb{N}^{*}}$.

Assumption 6.1. We suppose that $q_{n}$ and $K$ are such that the following hold:

(A.1) $G_{q_{n}}(n, K)$ converges almost surely and its limit is the graphon $K \in L^{\infty}\left(\Omega^{2}\right)$;

(A.2) $\sup _{n \geq 1} q_{n}<+\infty$.

Graph models that verify (A.1)-(A.2) are discussed in [29, Proposition 2.1]. They encompass the dense random graph model (i.e., with $\Theta\left(n^{2}\right)$ edges) extensively studied in [35, 10], for which $q_{n} \geq c>0$. This graph model allows also to generate sparse (but not too sparse) graph models; see [8]. That is graphs with $o\left(n^{2}\right)$ but $\omega(n)$ edges, i.e., that the average degree tends to infinity with $n$. For example, one can take $q_{n}=\exp \left(-\log (n)^{1-\delta}\right)=o(1)$, where $\left.\delta \in\right] 0,1[$.

\subsection{Networks on graphs generated by deterministic nodes}

In order to make our reasoning simpler, it will be convenient to assume first that the sequence $\mathbf{X}$ is deterministic. Capitalizing on this result, we will then deal with the totally random model (i.e.; generated by random nodes) in Section 6.2 by a simple marginalization argument combined with additional assumptions to get the convergence and quantify the corresponding rate. As we have mentioned before, we shall denote $\mathbf{x}=\left(\mathbf{x}_{1}, \cdots, \mathbf{x}_{n}\right)$ as we assume that the sequence of nodes is deterministic. Relying on this notation, we define the parameter $\delta(n)$ as the maximal size of the spacings of $\mathbf{x}$, i.e.,

$$
\delta(n)=\max _{i \in[n]}\left|\mathbf{x}_{(i)}-\mathbf{x}_{(i-1)}\right| .
$$

Next, we consider the discrete counterpart of $\left(\mathcal{V} \mathcal{P}^{\lambda, p}\right)$ on the graph $G_{n}$

$$
\min _{u_{n} \in \mathbb{R}^{n}}\left\{E_{n, \lambda}\left(u_{n}, g_{n}, K_{n}\right) \stackrel{\text { def }}{=} \frac{1}{2 \lambda n}\left\|u_{n}-g_{n}\right\|_{2}^{2}+\frac{1}{2 p n^{2}} \sum_{i, j=1}^{n} \Lambda_{n i j}\left|u_{n j}-u_{n i}\right|^{p}\right\}, \quad\left(\mathcal{V P}_{d, n}^{\lambda, p}\right)
$$

where

$$
g_{i}=\frac{1}{\left|\Omega_{n i}^{\mathbf{x}}\right|} \int_{\Omega_{n i}^{\mathbf{x}}} g(x) d x
$$

Theorem 6.1. Suppose that $p \in\left[1,2\left[, g \in L^{2}(\Omega)\right.\right.$ and $K$ is a nonnegative measurable, symmetric and bounded mapping. Let $u^{\star}$ and $u_{n}^{\star}$ be the unique minimizers of $\left(\mathcal{V} \mathcal{P}^{\lambda, p}\right)$ and $\left(\mathcal{V P} \mathcal{d}_{d, n}^{\lambda, p}\right)$, respectively. Let $p^{\prime}=\frac{2}{2-p}$. 
(i) There exist positive constants $C$ and $C_{1}$ that do not depend on $n$, such that for any $\beta>0$

$$
\begin{aligned}
\left\|I_{n} u_{n}^{\star}-u^{\star}\right\|_{L^{2}(\Omega)}^{2} & \leq C\left(\left(\beta \frac{\log (n)}{n}+\frac{1}{q_{n}^{\left(p^{\prime}-1\right)} n^{p^{\prime} / 2}}\right)^{1 / p^{\prime}}+\left\|g-I_{n} g_{n}\right\|_{L^{2}(\Omega)}^{2}\right. \\
& \left.+\left\|g-I_{n} g_{n}\right\|_{L^{2}(\Omega)}+\left\|K-I_{n} \hat{K}_{n}^{\mathbf{x}}\right\|_{L^{p^{\prime}\left(\Omega^{2}\right)}}+\left\|u^{\star}-I_{n} P_{n} u^{\star}\right\|_{L^{3-p}(\Omega)}\right),
\end{aligned}
$$

with probability at least $1-2 n^{-C_{1} q_{n}^{2 p^{\prime}-1} \beta}$.

(ii) Assume moreover that $g \in L^{\infty}(\Omega) \cap \operatorname{Lip}\left(s, L^{q}(\Omega)\right)$, with $\left.\left.s \in\right] 0,1\right]$ and $q \in[2 /(3-p), 2]$, that $K(x, y)=J(|x-y|), \forall(x, y) \in \Omega^{2}$, with $J$ a nonnegative bounded measurable mapping on $\Omega$, and $\left.\left.K \in \operatorname{Lip}\left(s^{\prime}, L^{q^{\prime}}\left(\Omega^{2}\right)\right),\left(s^{\prime}, q^{\prime}\right) \in\right] 0,1\right] \times\left[p^{\prime},+\infty\right]$ and $q_{n}\|K\|_{L^{\infty}\left(\Omega^{2}\right)} \leq 1$. Then there exist positive constants $C$ and $C_{1}$ that do not depend on $n$, such that for any $\beta>0$

$$
\left\|I_{n} u_{n}^{\star}-u^{\star}\right\|_{L^{2}(\Omega)}^{2} \leq C\left(\left(\beta \frac{\log (n)}{n}+\frac{1}{q_{n}^{\left(p^{\prime}-1\right)} n^{p^{\prime} / 2}}\right)^{1 / p^{\prime}}+\delta(n)^{-\min \left(s q / 2, s^{\prime}\right)}\right),
$$

with probability at least $1-2 n^{-C_{1} q_{n}^{2 p^{\prime}-1} \beta}$.

Before delving into the proof, some remarks are in order.

\section{Remark 6.1.}

(i) The first term in the bounds (58)-(59) can be replaced by

$$
\beta^{1 / p^{\prime}}\left(\frac{\log (n)}{n}\right)^{1 / p^{\prime}}+\frac{1}{q_{n}^{\left(1-1 / p^{\prime}\right)} n^{1 / 2}}
$$

(ii) The last term in the latter bound can be rewritten as

$$
n^{-1 / 2} q_{n}^{-\left(1-1 / p^{\prime}\right)}= \begin{cases}\left(q_{n} n\right)^{-1 / 2} & \text { if } p^{\prime}=2, \\ q_{n}^{1 / p^{\prime}}\left(q_{n}^{2} n\right)^{-1 / 2} & \text { if } p^{\prime}>2 .\end{cases}
$$

Thus, if $\inf _{n \geq 1} q_{n}>0$, as is the case when the graph is dense, then the term (60) is in the order of $n^{-1 / 2}$ with probability at least $1-n^{-c \beta}$ for some $c>0$. If $q_{n}$ is allowed to be o(1), i.e., sparse graphs, then (60) is o(1) if either $q_{n} n \rightarrow+\infty$ for $p^{\prime}=2$, or $q_{n}^{2} n \rightarrow+\infty$ for $p^{\prime}>2$. The probability of success is at least $1-e^{-C_{1} \beta \log (n)^{1-\delta}}$ provided that $q_{n}=\log (n)^{-\delta /\left(2 p^{\prime}-1\right)}$, with $\delta \in\left[0,1\left[\right.\right.$. All these conditions on $q_{n}$ are fulfilled by the inhomogenous graph model discussed above.

(iii) In fact, if $\inf _{n \geq 1} q_{n} \geq c>0$, then we have $\sum_{n \geq 1} n^{-C_{1} q_{n}^{2 p-1} \beta} \leq \sum_{n \geq 1} n^{-C_{1} c^{2 p-1} \beta}<+\infty$ provided that $\beta>\left(C_{1} c^{2 p-1}\right)^{-1}$. Thus, if this holds, invoking the (first) Borel-Cantelli lemma, it follows that the bounds of Theorem 6.1 hold almost surely. The same reasoning carries over for the bounds of Theorem 6.2. 
Proof : In the following $C$ is any positive constant independent of $n$.

(i) We start by arguing as in the proof of Theorem 4.1. Similarly to (19), we now have

$$
\begin{aligned}
\frac{1}{2 \lambda}\left\|I_{n} u_{n}^{\star}-u^{\star}\right\|_{L^{2}(\Omega)}^{2} \leq\left(E_{\lambda}\left(I_{n} u_{n}^{\star}, g, K\right)-E_{n, \lambda}\left(u_{n}^{\star}, g_{n}, \Lambda_{n}\right)\right) & \\
& -\left(E_{\lambda}\left(u^{\star}, g, K\right)-E_{n, \lambda}\left(u_{n}^{\star}, g_{n}, \Lambda_{n}\right)\right) .
\end{aligned}
$$

The first term can be bounded similarly to (21)-(22) to get

$$
\begin{aligned}
& E_{\lambda}\left(I_{n} u_{n}^{\star}, g, K\right)-E_{n, \lambda}\left(u_{n}^{\star}, g_{n}, \Lambda_{n}\right) \\
& \leq C\left(\left\|I_{n} g_{n}-g\right\|_{L^{2}(\Omega)}^{2}+\left\|I_{n} g_{n}-g\right\|_{L^{2}(\Omega)}\right. \\
& \left.\quad+\left|\int_{\Omega^{2}}\left(K(x, y)-I_{n} \Lambda_{n}(x, y)\right)\right| I_{n} u_{n}^{\star}(y)-\left.I_{n} u_{n}^{\star}(x)\right|^{p} d x d y \mid\right) \\
& \leq C\left(\left\|I_{n} g_{n}-g\right\|_{L^{2}(\Omega)}^{2}+\left\|I_{n} g_{n}-g\right\|_{L^{2}(\Omega)}\right. \\
& \quad+\left|\int_{\Omega^{2}}\left(K(x, y)-I_{n} \hat{K}_{n}^{\mathbf{x}}(x, y)\right)\right| I_{n} u_{n}^{\star}(y)-\left.I_{n} u_{n}^{\star}(x)\right|^{p} d x d y \mid \\
& \left.\quad+\left|\int_{\Omega^{2}}\left(I_{n} \hat{K}_{n}^{\mathbf{x}}(x, y)-I_{n} \Lambda_{n}(x, y)\right)\right| I_{n} u_{n}^{\star}(y)-\left.I_{n} u_{n}^{\star}(x)\right|^{p} d x d y \mid\right) .
\end{aligned}
$$

The second term in (62) is $O\left(\left\|K-I_{n} \hat{K}_{n}^{\mathbf{x}}\right\|_{L^{p^{\prime}}\left(\Omega^{2}\right)}\right)$, see (23). For the last term, we have using Jensen and Hölder inequalities,

$$
\begin{aligned}
& \left|\int_{\Omega^{2}}\left(I_{n} \hat{K}_{n}^{\mathbf{x}}(x, y)-I_{n} \Lambda_{n}(x, y)\right)\right| I_{n} u_{n}^{\star}(y)-\left.I_{n} u_{n}^{\star}(x)\right|^{p} d x d y \mid \\
& \leq 2^{p-1}\left(\int_{\Omega}\left|\int_{\Omega}\left(I_{n} \hat{K}_{n}^{\mathbf{x}}(x, y)-I_{n} \Lambda_{n}(x, y)\right) d y\right|\left|I_{n} u_{n}^{\star}(x)\right|^{p} d x\right. \\
& \left.\quad+\int_{\Omega}\left|\int_{\Omega}\left(I_{n} \hat{K}_{n}^{\mathbf{x}}(x, y)-I_{n} \Lambda_{n}(x, y)\right) d x\right|\left|I_{n} u_{n}^{\star}(y)\right|^{p} d y\right) \\
& \leq C\left(\int_{\Omega}\left|\int_{\Omega}\left(I_{n} \hat{K}_{n}^{\mathbf{x}}(x, y)-I_{n} \Lambda_{n}(x, y)\right) d y\right|^{p^{\prime}} d x\right)^{1 / p^{\prime}} \\
& \left.\quad+\left(\int_{\Omega}\left|\int_{\Omega}\left(I_{n} \hat{K}_{n}^{\mathbf{x}}(x, y)-I_{n} \Lambda_{n}(x, y)\right) d x\right|^{p^{\prime}} d y\right)^{1 / p^{\prime}}\right) \\
& =C\left(\left\|Z_{n}\right\|_{p^{\prime}, n}+\left\|W_{n}\right\|_{p^{\prime}, n}\right),
\end{aligned}
$$

where

$$
Z_{n i} \stackrel{\text { def }}{=} \frac{1}{n} \sum_{j=1}^{n}\left(\stackrel{\wedge}{K}_{n i j}^{\mathbf{x}}-\Lambda_{n i j}\right) \text { and } W_{n j} \stackrel{\text { def }}{=} \frac{1}{n} \sum_{i=1}^{n}\left(\stackrel{\wedge}{K}_{n i j}^{\mathbf{x}}-\Lambda_{n i j}\right)
$$


By virtue of [29, Lemma A.1], together with (A.2) and the fact that $p^{\prime} \geq 2$, there exists a positive constant $C_{1}$, such that for any $\beta>0$

$$
\mathbb{P}\left(\left\|Z_{n}\right\|_{p^{\prime}, n} \geq \varepsilon\right) \leq n^{-C_{1} q_{n}^{2 p^{\prime}-1} \beta}
$$

with

$$
\varepsilon=\left(\beta \frac{\log (n)}{n}+\frac{1}{q_{n}^{\left(p^{\prime}-1\right)} n^{p^{\prime} / 2}}\right)^{1 / p^{\prime}} .
$$

The same bound also holds for $\left\|W_{n}\right\|_{p^{\prime}, n}$. A union bound then leads to

$$
\left\|Z_{n}\right\|_{p^{\prime}, n}+\left\|W_{n}\right\|_{p^{\prime}, n} \leq 2 \varepsilon
$$

with probability at least $1-2 n^{-C_{1} q_{n}^{2 p^{\prime}-1} \beta}$.

Let us now turn to the second term in (61). Using (8) and the fact that $u_{n}^{\star}$ is the unique minimizer of $\left(\mathcal{V} \mathcal{P}_{d, n}^{\lambda, p}\right)$, we have

$$
\begin{aligned}
E_{\lambda}\left(I_{n} u_{n}^{\star}, I_{n} g_{n}, I_{n} \Lambda_{n}\right)-E_{\lambda}\left(u^{\star}, g, K\right) & \leq\left(R_{p}\left(I_{n} P_{n} u^{\star}, K\right)-R_{p}\left(u^{\star}, K\right)\right) \\
& +\left(R_{p}\left(I_{n} P_{n} u^{\star}, I_{n} \hat{K}_{n}^{\mathbf{x}}\right)-R_{p}\left(I_{n} P_{n} u^{\star}, K\right)\right) \\
& +\left(R_{p}\left(I_{n} P_{n} u^{\star}, I_{n} \Lambda_{n}\right)-R_{p}\left(I_{n} P_{n} u^{\star}, I_{n} \hat{K}_{n}^{\mathbf{x}}\right)\right) .
\end{aligned}
$$

The first term is bounded as in (26), which yields

$$
\left|R_{p}\left(I_{n} P_{n} u^{\star}, K\right)-R_{p}\left(u^{\star}, K\right)\right| \leq C\left\|u^{\star}-I_{n} P_{n} u^{\star}\right\|_{L^{\frac{2}{3-p}}(\Omega)} .
$$

The second term follows from (27)

$$
\left|R_{p}\left(I_{n} P_{n} u^{\star}, I_{n} \stackrel{\wedge}{\mathbf{x}}_{n}^{\mathbf{x}}\right)-R_{p}\left(I_{n} P_{n} u^{\star}, K\right)\right| \leq C\left\|K-I_{n} \stackrel{\wedge}{K}_{n}^{\mathbf{x}}\right\|_{L^{p^{\prime}}\left(\Omega^{2}\right)}
$$

The last term is upper-bounded exactly as in (63) and (65).

Inserting (62), (63), (65), (66), (67) and (68) into (61), we get the claimed bound.

(ii) Insert (45) and (51) into (58) after replacing $1 / n$ by $\delta(n)$.

\subsection{Networks on graphs generated by random nodes}

Let us turn now to the totally random model. The discrete counterpart of $\left(\mathcal{V} \mathcal{P}^{\lambda, p}\right)$ on the totally random sequence of graphs $\left\{G_{q_{n}}\right\}_{n \in \mathbb{N}^{*}}$ is given by

$$
\min _{u_{n} \in \mathbb{R}^{n}}\left\{E_{n, \lambda}\left(u_{n}, g_{n}, K_{n}\right) \stackrel{\text { def }}{=} \frac{1}{2 \lambda n}\left\|u_{n}-g_{n}\right\|_{2}^{2}+\frac{1}{n^{2}} \sum_{i, j=1}^{n} \Upsilon_{i j}\left|u_{n j}-u_{n i}\right|^{p}\right\}, \quad \quad\left(\mathcal{V} \mathcal{P}_{r, n}^{\lambda, p}\right)
$$


where we recall that the random variables $\Upsilon_{i j}$ are independent with $q_{n} \Upsilon_{i j}$ following the Bernoulli distribution with parameter $\mathbb{E}\left(q_{n} \hat{K}_{n i j}^{\mathbf{X}}\right)$ defined above.

Observe that for the totally random model, $\delta(n)$ is a random variable. Thus, we have to derive a bound on it. In [29, Lemma 3.2], it was shown that

$$
\delta(n) \leq t \frac{\log (n)}{n}
$$

with probability at least $1-n^{-t}$, where $\left.t \in\right] 0, e[$.

Combining this bound with Theorem 6.1 (after conditioning and integrating) applied to the totally random sequence $\left\{G_{q_{n}}\right\}_{n \in \mathbb{N}^{*}}$, we get the following result.

Theorem 6.2. Suppose that $p \in\left[1,2\left[, g \in L^{2}(\Omega)\right.\right.$ and $K$ is a nonnegative measurable, symmetric and bounded mapping. Let $u^{\star}$ and $u_{n}^{\star}$ be the unique minimizers of $\left(\mathcal{V} \mathcal{P}^{\lambda, p}\right)$ and $\left(\mathcal{V} \mathcal{P}_{r, n}^{\lambda, p}\right)$, respectively. Let $p^{\prime}=\frac{2}{2-p}$.

(i) There exist positive constants $C$ and $C_{1}$ that do not depend on $n$, such that for any $\beta>0$

$$
\begin{aligned}
\left\|I_{n} u_{n}^{\star}-u^{\star}\right\|_{L^{2}(\Omega)}^{2} & \leq C\left(\left(\beta \frac{\log (n)}{n}+\frac{1}{q_{n}^{\left(p^{\prime}-1\right)} n^{p^{\prime} / 2}}\right)^{1 / p^{\prime}}+\left\|g-I_{n} g_{n}\right\|_{L^{2}(\Omega)}^{2}\right. \\
& \left.+\left\|g-I_{n} g_{n}\right\|_{L^{2}(\Omega)}+\left\|K-I_{n} \hat{K}_{n}^{\mathbf{X}}\right\|_{L^{p^{\prime}}\left(\Omega^{2}\right)}+\left\|u^{\star}-I_{n} P_{n} u^{\star}\right\|_{L^{3-p}(\Omega)}\right),
\end{aligned}
$$

with probability at least $1-2 n^{-C_{1} q_{n}^{2 p^{\prime}-1} \beta}$.

(ii) Assume moreover that $g \in L^{\infty}(\Omega) \cap \operatorname{Lip}\left(s, L^{q}(\Omega)\right)$, with $\left.\left.s \in\right] 0,1\right]$ and $q \in[2 /(3-p)$, 2$]$, that $K(x, y)=J(|x-y|), \forall(x, y) \in \Omega^{2}$, with $J$ a nonnegative bounded measurable mapping on $\Omega$, that $\left.\left.K \in \operatorname{Lip}\left(s^{\prime}, L^{q^{\prime}}\left(\Omega^{2}\right)\right),\left(s^{\prime}, q^{\prime}\right) \in\right] 0,1\right] \times\left[p^{\prime},+\infty\right]$ and $q_{n}\|K\|_{L^{\infty}\left(\Omega^{2}\right)} \leq 1$. Then there exist positive constants $C$ and $C_{1}$ that do not depend on $n$, such that for any $\beta>0$ and $\left.t \in\right] 0, e[$

$$
\left\|I_{n} u_{n}^{\star}-u^{\star}\right\|_{L^{2}(\Omega)}^{2} \leq C\left(\left(\beta \frac{\log (n)}{n}+\frac{1}{q_{n}^{\left(p^{\prime}-1\right)} n^{p^{\prime} / 2}}\right)^{1 / p^{\prime}}+\left(t \frac{\log (n)}{n}\right)^{\min \left(s q / 2, s^{\prime}\right)}\right)
$$

with probability at least $1-\left(2 n^{-C_{1} q_{n}^{2 p^{\prime}-1} \beta}+n^{-t}\right)$.

Proof : Again, $C$ will be any positive constant independent of $n$.

(i) Let

$$
\begin{aligned}
\varepsilon^{\prime}=C & \left(\left(\beta \frac{\log (n)}{n}+C \frac{1}{q_{n}^{\left(p^{\prime}-1\right)} n^{p^{\prime} / 2}}\right)^{1 / p^{\prime}}+\left\|g-I_{n} g_{n}\right\|_{L^{2}(\Omega)}^{2}+\left\|g-I_{n} g_{n}\right\|_{L^{2}(\Omega)}\right. \\
& \left.+\left\|K-I_{n} \hat{K}_{n}^{\mathbf{X}}\right\|_{L^{p^{\prime}\left(\Omega^{2}\right)}}+\left\|u^{\star}-I_{n} P_{n} u^{\star}\right\|_{L^{3-p}(\Omega)}\right) .
\end{aligned}
$$


Using (58), and independence of this bound from $\mathbf{x}$, we have

$$
\begin{aligned}
\mathbb{P}\left(\left\|I_{n} u_{n}^{\star}-u^{\star}\right\|_{L^{2}(\Omega)}^{2} \geq \varepsilon^{\prime}\right) & =\frac{1}{|\Omega|^{n}} \int_{\Omega^{n}} \mathbb{P}\left(\left\|I_{n} u_{n}^{\star}-u^{\star}\right\|_{L^{2}(\Omega)}^{2} \geq \varepsilon^{\prime} \mid \mathbf{X}=\mathbf{x}\right) d \mathbf{x} \\
& \leq \frac{1}{|\Omega|^{n}} \int_{\Omega^{n}} 2 n^{-C_{1} q_{n}^{2 p^{\prime}-1} \beta} d \mathbf{x} \\
& =2 n^{-C_{1} q_{n}^{2 p^{\prime}-1} \beta} .
\end{aligned}
$$

(ii) Recall $\varepsilon$ in $(64)$ and $\kappa=C\left(t \frac{\log (n)}{n}\right)^{\min \left(s q / 2, s^{\prime}\right)}$. Denote the event

$A_{1}:\left\{\left\|g-I_{n} g_{n}\right\|_{L^{2}(\Omega)}^{2}+\left\|g-I_{n} g_{n}\right\|_{L^{2}(\Omega)}+\left\|K-I_{n} \stackrel{\wedge}{K}_{n}^{\mathbf{X}}\right\|_{L^{p^{\prime}\left(\Omega^{2}\right)}}+\left\|u^{\star}-I_{n} P_{n} u^{\star}\right\|_{L^{\frac{2}{3-p}}(\Omega)} \leq \kappa\right\}$.

In view of (45), (51) and (69), and that under our assumptions $\hat{K}_{n}^{\mathbf{X}}=K_{n}^{\mathbf{X}}$, we have

$$
\mathbb{P}\left(A_{1}\right) \geq \mathbb{P}\left(\delta(n) \leq t \frac{\log (n)}{n}\right) \geq 1-n^{-t}
$$

Let the event

$$
A_{2}:\left\{\left\|Z_{n}\right\|_{p^{\prime}, n}+\left\|W_{n}\right\|_{p^{\prime}, n} \leq 2 \varepsilon\right\}
$$

and denote $A_{i}^{c}$ the complement of the event $A_{i}$. It then follows from (65) and the union bound that

$$
\begin{aligned}
\mathbb{P}\left(\left\|I_{n} u_{n}^{\star}-u^{\star}\right\|_{L^{2}(\Omega)}^{2} \leq 2 C \varepsilon+\kappa\right) & \geq \mathbb{P}\left(A_{1} \cap A_{2}\right)=1-\mathbb{P}\left(A_{1}^{c} \cup A_{2}^{c}\right) \\
& \geq 1-\sum_{i=1}^{2} \mathbb{P}\left(A_{i}^{c}\right) \geq 1-\left(2 n^{-C_{1} q_{n}^{2 p^{\prime}-1} \beta}+n^{-t}\right)
\end{aligned}
$$

which leads to the claimed result.

When $p=1$ (i.e., nonlocal total variation), $g \in L^{\infty}(\Omega) \cap \operatorname{Lip}\left(s, L^{2}(\Omega)\right)$ and $K$ is a sufficiently smooth function, one can deduce from Theorem 6.2 that with high probability, the solution to the discrete problem $\left(\mathcal{V} \mathcal{P}_{r, n}^{\lambda, p}\right)$ converges to that of the continuum problem $\left(\mathcal{V} \mathcal{P}^{\lambda, p}\right)$ at the rate $O\left(\left(\frac{\log (n)}{n}\right)^{-\min (1 / 2, s)}\right)$. Compared to the deterministic graph model, there is overhead due to the randomness of the graph model which is captured in the rate and the extra-logarithmic factor.

\section{Numerical results}

In this section, we will apply the variational regularization problem $\left(\mathcal{V} \mathcal{P}_{n}^{\lambda, p}\right)$ to a few applications, and illustrate numerically our bounds. 


\subsection{Minimization algorithm}

The algorithm we will describe in this subsection is valid for any $p \in[1,+\infty]^{1}$. The minimization problem $\left(\mathcal{V P}_{n}^{\lambda, p}\right)$ can be rewritten in the following form

$$
\min _{u_{n} \in \mathbb{R}^{n}} \frac{1}{2}\left\|u_{n}-g_{n}\right\|_{2}^{2}+\frac{\lambda_{n}}{p}\left\|\nabla_{K_{n}} u_{n}\right\|_{p}^{p},
$$

where $\lambda_{n}=\lambda /(2 n), \nabla_{K_{n}}$ is the (nonlocal) weighted gradient operator with weights $K_{n i j}$, defined as

$$
\begin{aligned}
\nabla_{K_{n}}: \mathbb{R}^{n} & \rightarrow \mathbb{R}^{n \times n} \\
u_{n} & \mapsto V_{n}, \quad V_{n i j}=K_{n i j}^{1 / p}\left(u_{n j}-u_{n i}\right), \quad \forall(i, j) \in[n]^{2} .
\end{aligned}
$$

This is a linear operator whose adjoint, the (nonlocal) weighted divergence operator denoted $\operatorname{div}_{K_{n}}$. It is easy to show that

$$
\begin{aligned}
& \operatorname{div}_{K_{n}}: \mathbb{R}^{n \times n} \rightarrow \mathbb{R}^{n} \\
& V_{n} \mapsto u_{n}, \quad u_{n i}=\sum_{m=1}^{n} K_{n m i}^{1 / p} V_{n m i}-\sum_{j=1}^{n} K_{n i j}^{1 / p} V_{n i j}, \quad \forall i \in[n] .
\end{aligned}
$$

Problem (72) can be easily solved using standard duality-based first-order algorithms. For this we follow [23].

By standard conjugacy calculus, the Fenchel-Rockafellar dual problem of (72) reads

$$
\min _{V_{n} \in \mathbb{R}^{n \times n}} \frac{1}{2}\left\|g_{n}-\operatorname{div}_{K_{n}} V_{n}\right\|_{2}^{2}+\frac{\lambda_{n}}{q}\left\|V_{n} / \lambda_{n}\right\|_{q}^{q},
$$

where $q$ is the Hölder dual of $p$, i.e. $1 / p+1 / q=1$. One can show with standard arguments that the dual problem (73) has a convex compact set of minimizers for any $p \in[1,+\infty[$. Moreover, the unique solution $u_{n}^{\star}$ to the primal problem (72) can be recovered from any dual solution $V_{n}^{\star}$ as

$$
u_{n}^{\star}=g_{n}-\operatorname{div}_{K_{n}} V_{n}^{\star} .
$$

It remains now to solve (73). The latter can be solved with the (accelerated) FISTA iterative scheme $[38,4,15]$ which reads in this case

$$
\begin{aligned}
W_{n}^{k} & =V_{n}^{k}+\frac{k-1}{k+b}\left(V_{n}^{k}-V_{n}^{k-1}\right) \\
V_{n}^{k+1} & =\operatorname{prox}_{\gamma \frac{\lambda_{n}}{q}\left\|\cdot / \lambda_{n}\right\|_{q}^{q}}\left(W_{n}^{k}+\gamma \nabla_{K_{n}}\left(g_{n}-\operatorname{div}_{K_{n}}\left(W_{n}^{k}\right)\right)\right) \\
u_{n}^{k+1} & =g_{n}-\operatorname{div}_{K_{n}} V_{n}^{k+1},
\end{aligned}
$$

where $\left.\gamma \in] 0,\left(\sup _{\left\|u_{n}\right\|_{2}=1}\left\|\nabla_{K_{n}} u_{n}\right\|_{2}\right)^{-1}\right], b>2$, and we recall that prox ${ }_{\tau F}$ is the proximal mapping of the proper lsc convex function $F$ with $\tau>0$, i.e.,

$$
\operatorname{prox}_{\tau F}(W)=\underset{V \in \mathbb{R}^{n \times n}}{\operatorname{Argmin}} \frac{1}{2}\|V-W\|_{2}^{2}+\tau F(V) .
$$

The convergence guarantees of scheme (74) are summarized in the following proposition.

\footnotetext{
${ }^{1}$ Obviously $\lim _{p \rightarrow+\infty} \frac{1}{p}\|\cdot\|_{p}^{p}=\iota_{\left\{u_{n} \in \mathbb{R}^{n}:\left\|u_{n}\right\|_{\infty} \leq 1\right\}}$.
} 
Proposition 7.1. The primal iterates $u_{n}^{k}$ converge to $u_{n}^{\star}$, the unique minimizer of $\left(\mathcal{V P} \mathcal{P}_{n}^{\lambda, p}\right)$, at the rate

$$
\left\|u_{n}^{k}-u_{n}^{\star}\right\|_{2}=o(1 / k) .
$$

Proof : Combine [23, Theorem 2] and [2, Theorem 1.1].

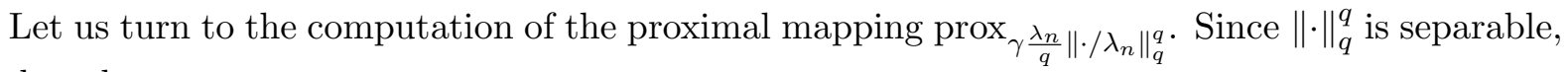
one has that

$$
\operatorname{prox}_{\gamma \frac{\lambda_{n}}{q}\left\|\cdot / \lambda_{n}\right\|_{q}^{q}}(W)=\left(\operatorname{prox}_{\gamma \frac{\lambda_{n}}{q}\left|\cdot / \lambda_{n}\right|^{q}}\left(W_{i j}\right)\right)_{(i, j) \in[n]^{2}} .
$$

Moreover, as $|\cdot|^{q}$ is an even function on $\mathbb{R}, \operatorname{prox}_{\gamma \frac{\lambda_{n}}{q}\left|\cdot / \lambda_{n}\right|^{q}}$ is an odd mapping on $\mathbb{R}$, that is,

$$
\operatorname{prox}_{\gamma \frac{\lambda_{n}}{q}\left|\cdot / \lambda_{n}\right|^{q}}\left(W_{i j}\right)=\operatorname{prox}_{\gamma \frac{\lambda_{n}}{q}\left|\cdot / \lambda_{n}\right|^{q}}\left(\left|W_{i j}\right|\right) \operatorname{sign}\left(W_{i j}\right) .
$$

In a nutshell, one has to compute $\operatorname{prox}_{\gamma \frac{\lambda_{n}}{q}\left|\cdot / \lambda_{n}\right|^{q}}(t)$ for $t \in \mathbb{R}^{+}$. We distinguish different situations depending on the value of $q$ :

- $q=+\infty$ (i.e., $p=1$ ): this case amounts to computing the orthogonal projector on $\left[-\lambda_{n}, \lambda_{n}\right]$, which reads

$$
t \in \mathbb{R}^{+} \mapsto \operatorname{proj}_{\left[-\lambda_{n}, \lambda_{n}\right]}(t)=\min \left(t, \lambda_{n}\right) .
$$

- $q=1$ (i.e., $p=+\infty$ ): this case corresponds to the well-known soft-thresholding operator, which is given by

$$
t \in \mathbb{R}^{+} \mapsto \operatorname{prox}_{\gamma|\cdot|}(t)=\max (t-\gamma, 0)
$$

- $q=2$ (i.e., $p=2$ ): it is immediate to see that

$$
\operatorname{prox}_{\gamma /\left(2 \lambda_{n}\right)|\cdot|^{2}}(t)=\frac{t}{1+\gamma / \lambda_{n}}
$$

- $q \in] 1,+\infty\left[\right.$ : in this case, as $|\cdot|^{q}$ is differentiable, the proximal point $\operatorname{prox}_{\gamma \frac{\lambda_{n}}{q}\left|\cdot / \lambda_{n}\right|^{q}}(t)$ is the unique solution $\alpha^{\star}$ on $\mathbb{R}^{+}$of the non-linear equation

$$
\alpha-t+\gamma \alpha^{p-1} / \lambda_{n}=0
$$

\subsection{Experimental setup}

We apply the scheme (74) to solve (72) in two applicative settings with nonlocal regularization on (weighted) graphs. The first one pertains to denoising of a function defined on a $2 \mathrm{D}$ point cloud, and the second one to signal denoising. In the first setting, the nodes of the graph are the points in the cloud and $u_{n i}$ is the value of point/vertex index $i$. For signal denoising, each graph node correspond to a signal sample, and $u_{n i}$ is the signal value at node/sample index $i$. We chose the nearest neighbour graph with the standard weighting kernel $e^{-|\mathbf{x}-\mathbf{y}|}$ when $|\mathbf{x}-\mathbf{y}| \leq \delta$ and 0 otherwise, where $\mathbf{x}$ and $\mathbf{y}$ are the $2 \mathrm{D}$ spatial coordinates of the points for the point cloud $^{2}$, and sample index for the signal case.

\footnotetext{
${ }^{2}$ For the $2 \mathrm{D}$ case, $(\mathbf{x}, \mathbf{y})$ are not to be confused with the "coordinates" $(x, y)$ of the graphon on the continuum, though there is a bijection from one to another.
} 


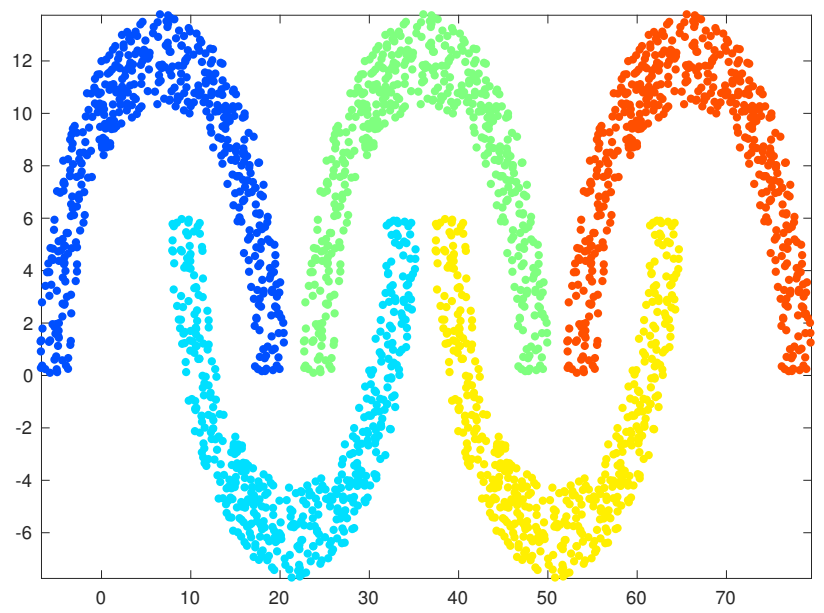

Figure 1: Original point cloud with $N=2500$ points.

Application to point cloud denoising The original point cloud used in our numerical experiments is shown in Figure 1. It consists of $N=2500$ points that are not on a regular grid. The function on this point cloud, denoted $u_{N}^{0}$, is piecewise-constant taking 5 values (5 clusters) in [5]. A noisy observation $g_{N}$ (see Figure 2(a)) is then generated by adding a white Gaussian noise of standard deviation 0.5 to $u_{N}^{0}$. Given the piecewise-constancy of $u_{N}^{0}$, we solved (72) with the natural choice $p=1$. The result is shown in Figure 2(b). Figure 2(c) displays the evolution of $\left\|u_{N}^{k}-u_{N}^{\star}\right\|_{2}$ as a function of the iteration counter $k$, which confirms the theoretical rate $o(1 / k)$ predicted above.

To illustrate our consistency results, $u^{\star}$ is needed while it is not known in our case. Therefore, we argue as follows. We consider the continuum extension of $I_{N} u_{N}^{\star}$ as a reference and compute $\| I_{n} u_{n}^{\star}-$ $I_{N} u_{N}^{\star} \|_{L^{2}(\Omega)}$ for varying $n \ll N$. By the triangle inequality, $\left\|I_{n} u_{n}^{\star}-I_{N} u_{N}^{\star}\right\|_{L^{2}(\Omega)}$ is clearly dominated by $\left\|I_{n} u_{n}^{\star}-u^{\star}\right\|_{L^{2}(\Omega)}$. Thus, for each value of $n \in[100, N / 8], n$ nodes are drawn uniformly at random in $[N]$ and $g_{n}$ is generated, which is a sampled version of $g_{N}$ at those nodes. This is replicated 20 times. For each replication, we solve (72) with $g_{n}$ and the same regularization parameter $\lambda$, and we compute the mean across the 20 replications of the squared-error $\left\|I_{n} u_{n}^{\star}-I_{N} u_{N}^{\star}\right\|_{L^{2}(\Omega)}^{2}$. The result is depicted in Figure 2(d). The gray-shaded area corresponds to one standard deviation of the error over the 20 replications. One indeed observes that the average error decreases at a rate consistent with the $O\left(n^{-1 / 2}\right)$ predicted by our results (see discussion after Theorem 5.2 with $s=1 / 2$ ).

Application to signal denoising In this experiment, we choose a piecewise-constant signal shown in Figure 3(a) for $N=1000$ together with its noisy version $g_{N}$ with additive white Gaussian noise of standard deviation 0.05 . Figure $3(\mathrm{~b})$ depicts the denoised signal $u_{N}^{\star}$ by solving (72) with $p=1$ and hand-tuned $\lambda$. Figure 3(c) also confirms the $o(1 / k)$ rate predicted above on $\left\|u_{N}^{k}-u_{N}^{\star}\right\|_{2}$.

We now illustrate the consistency bound result on a random sequence of graphs $\left\{G_{q_{n}}(n, K)\right\}_{n \in[100, N / 4]}$ generated according to Definition 6.1 with $q_{n}=1$. For each value of $n \in[100, N / 4], n$ nodes are drawn uniformly at random in $[N]$, and $g_{n}$ is generated, which is a sampled version of $g_{N}$ at those nodes. $n^{2}$ independent Bernoulli variables $\Lambda_{n i j}$ each with parameter $K_{n i j}$ are also generated. This is replicated 20 times. For each replication, we solve (72) with $g_{n}$ and the same regularization parameter $\lambda$, and we compute the mean across the 20 replications 
(a)

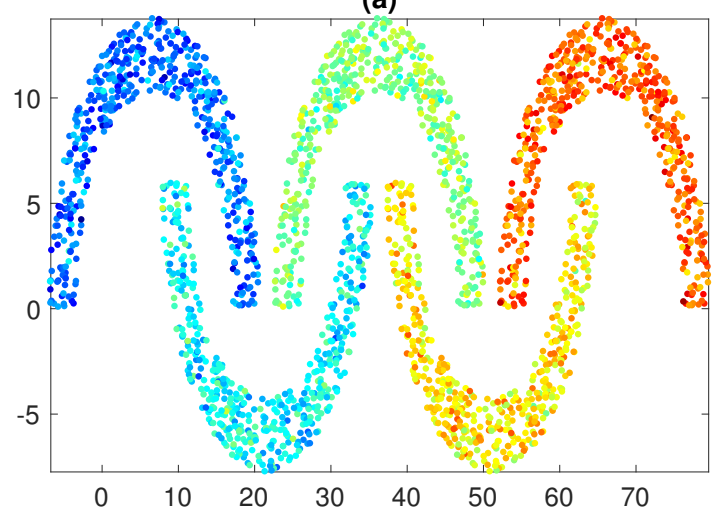

(c)

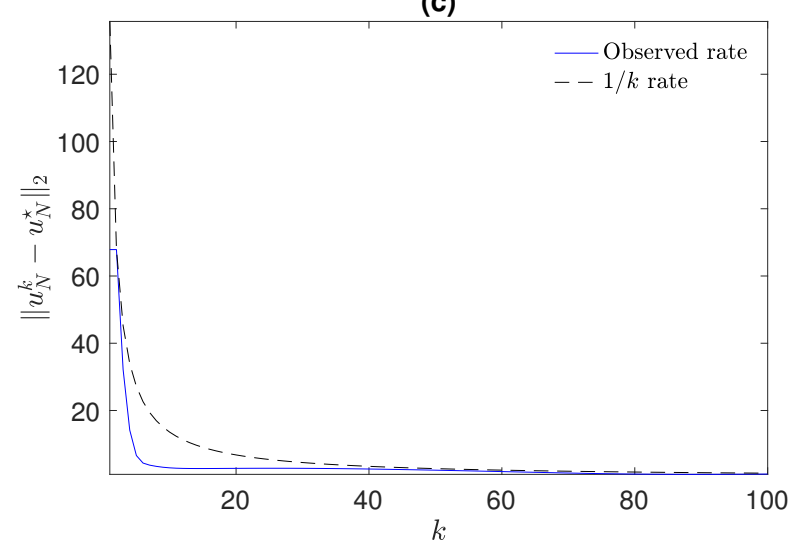

(b)

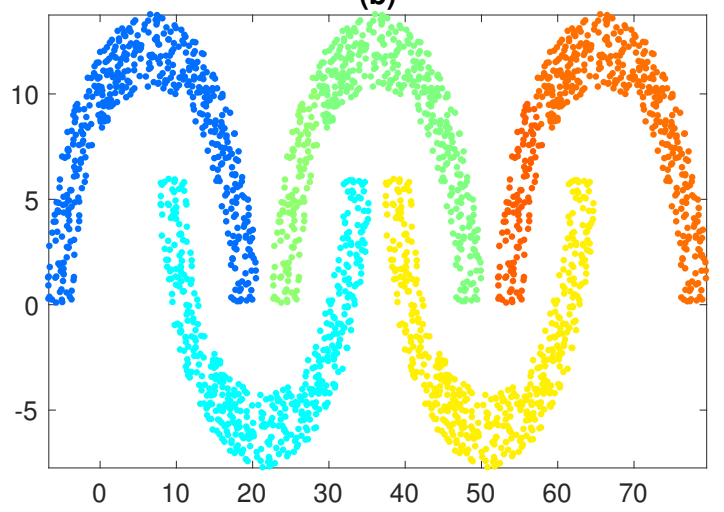

(d)

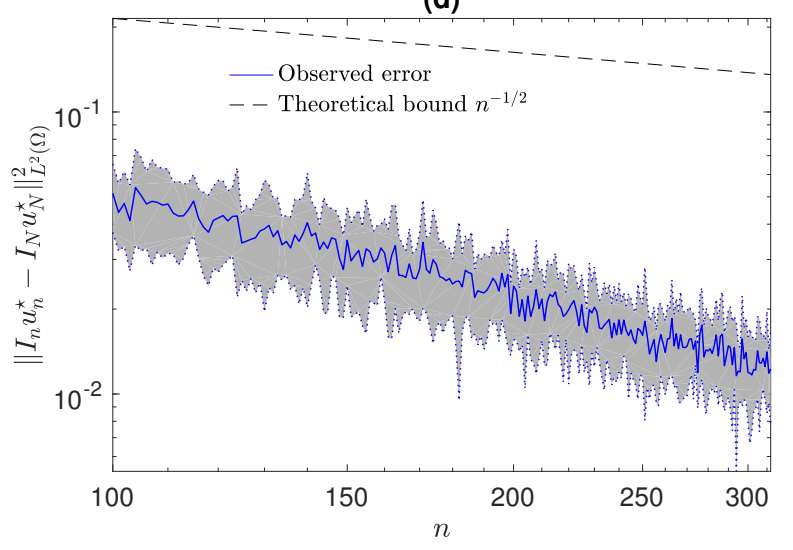

Figure 2: Results for point cloud denoising with $p=1$. (a) Noisy point cloud. (b) Recovered point cloud by solving (72). (c) Primal convergence criterion $\left\|u_{n}^{k}-u_{n}^{\star}\right\|_{2}$ as a function of the iteration counter $k$. (d) Mean error $\left\|I_{n} u_{n}^{\star}-I_{N} u_{N}^{\star}\right\|_{L^{2}(\Omega)}^{2}$ across replications as a function of $n$.

of the squared-error $\left\|I_{n} u_{n}^{\star}-I_{N} u_{N}^{\star}\right\|_{L^{2}(\Omega)}^{2}$. The result is reported in Figure 3(d). The gray-shaded area indicates one standard deviation of the error over the 20 replications. Again, the average error decreases in agreement with the rate $O\left((\log (n) / n)^{1 / 2}\right)$ predicted by Theorem 6.2 .

Acknowledgements This work was supported by the ANR grant GRAPHSIP. JF was partly supported by Institut Universitaire de France. JF and AE would like to acknowledge support within the EU grant No. 777826, the NoMADs project.

\section{References}

[1] F. Andreu-Vaillo, J. M. Mazón, J. D. Rossi, and J. J. Toledo-Melero. Nonlocal diffusion problems, volume 165 of Mathematical Surveys and Monographs. American Mathematical Society, 2010. 
(a)

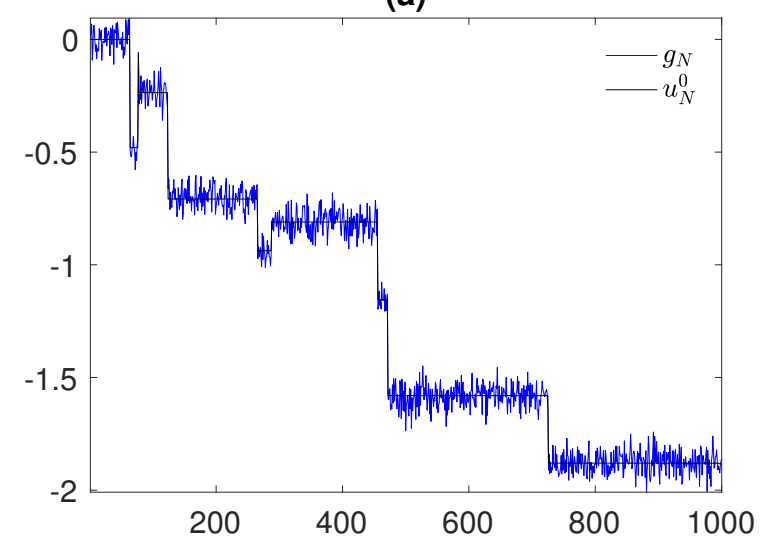

(c)

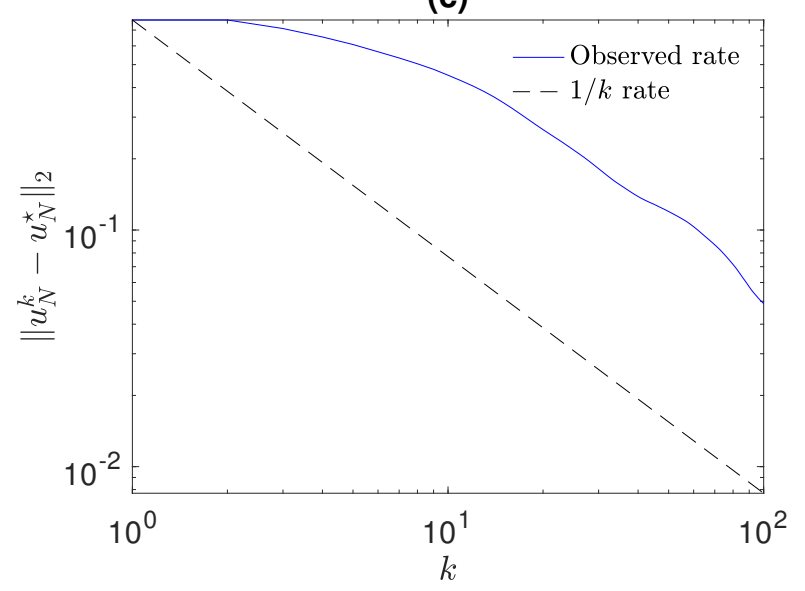

(b)

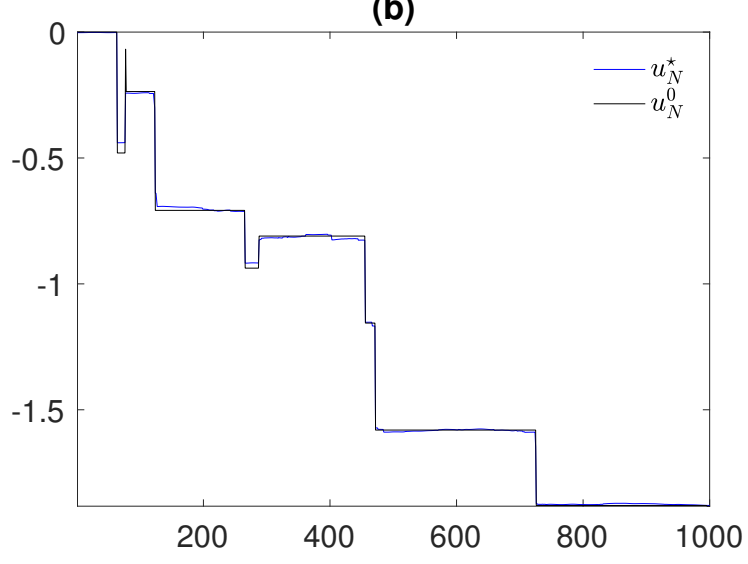

(d)

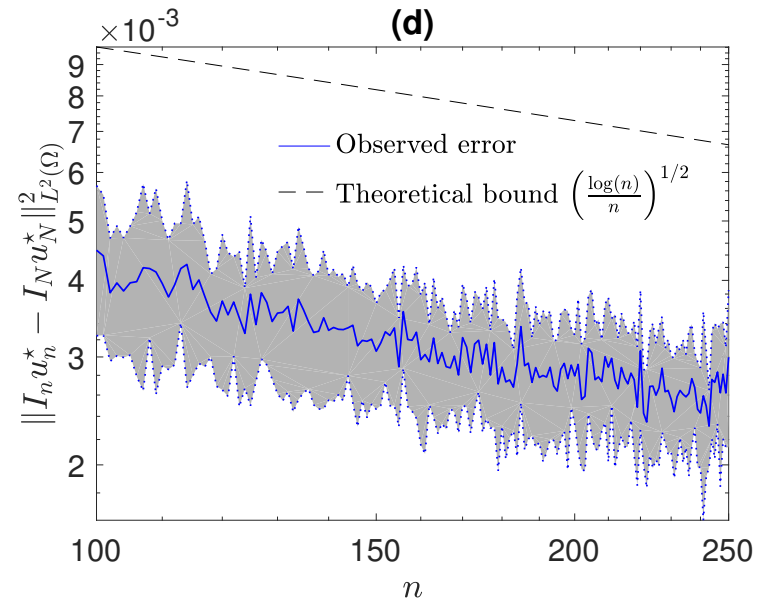

Figure 3: Results for signal denoising with $p=1$. (a) Noisy and original signal. (b) Denoised and original signal foor $N=1000$. (c) Primal convergence criterion $\left\|u_{n}^{k}-u_{n}^{\star}\right\|_{2}$ as a function of the iteration counter $k$. (d) Mean error $\left\|I_{n} u_{n}^{\star}-I_{N} u_{N}^{\star}\right\|_{L^{2}(\Omega)}^{2}$ as a function of $n$.

[2] H. Attouch and J. Peypouquet. The rate of convergence of Nesterov's accelerated ForwardBackward method is actually $o\left(k^{-2}\right)$. SIAM Journal on Optimization, 26(3):1824-1834, 2016.

[3] H. Bauschke and P. L. Combettes. Convex Analysis and Monotone Operator Theory in Hilbert Spaces. Springer, 2011.

[4] A. Beck and M. Teboulle. A fast iterative shrinkage-thresholding algorithm for linear inverse problems. SIAM Journal on Imaging Sciences, 2(1):183-202, 2009.

[5] S. J. Béla Bollobás and O. Reordan. The phase transition in inhomogeneous random graphs. arXiv:math/0504589v3 [math.PR], 2006.

[6] M. Belkin and P. Niyogi. Convergence of Laplacian eigenmaps. In Advances in Neural Information Processing Systems (NIPS), pages 129-136, 2007. 
[7] A. L. Bertozzi and A. Flenner. Diffuse interface models on graphs for analysis of high dimensional data. SIAM J. Multiscale Modeling and Simulation, 10(3):1090-1118, 2012.

[8] B. Bollobás and O. Riordan. Metrics for sparse graphs. arXiv:0708.1919v3 [math.CO], 2009.

[9] C. Borgs, J. Chayes, L. Lovász, V. Sós, and K. Vesztergombi. Limits of randomly grown graph sequences. European Journal of Combinatorics, 32(7):985 - 999, 2011.

[10] C. Borgs, J. Chayes, L. Lovász, V. Sós, and K. Vesztergombi. Limits of randomly grown graph sequences. European Journal of Combinatorics, 32(7):985 - 999, 2011.

[11] A. Buades, B. Coll, J. michel Morel, and C. Sbert. Non local demosaicing, 2007.

[12] A. Buades, B. Coll, and J. M. Morel. On image denoising methods. SIAM Multiscale Modeling and Simulation, 4(2):490-530, 2005.

[13] T. Bühler and M. Hein. Spectral clustering based on the graph p-Laplacian. In Proceedings of the 26th Annual International Conference on Machine Learning, pages 81-88, 2009.

[14] J. Calder. Consistency of lipschitz learning with infinite unlabeled data and finite labeled data. arXiv:1710.10364, 102017.

[15] A. Chambolle and C. Dossal. On the convergence of the iterates of the "fast iterative shrinkage/thresholding algorithm". Journal of Optimization Theory and Applications, 166(3):968$982,2015$.

[16] R. R. Coifman and S. Lafon. Diffusion maps. Appl. Comput. Harmon. Anal, 21(1):5-30, 2006.

[17] R. R. Coifman, S. Lafon, A. B. Lee, M. Maggioni, B. Nadler, F. Warner, and S. W. Zucker. Geometric diffusions as a tool for harmonic analysis and structure definition of data: Diffusion maps. Proceedings of the National Academy of Sciences, 102(21):7426-7431, 2005.

[18] R. A. DeVore and G. G. Lorentz. Constructive Approximation, volume 303 of Grundlehren der mathematischen. Springer-Verlag Berlin Heidelberg, 1993.

[19] A. El Alaoui, X. Cheng, A. Ramdas, M. J. Wainwright, and M. I. Jordan. Asymptotic behavior of $\ell_{p}$-based laplacian regularization in semi-supervised learning. In 29th Annual Conference on Learning Theory, pages 879-906, 2016.

[20] A. Elmoataz, O. Lezoray, and S. Bougleux. Nonlocal Discrete Regularization on Weighted Graphs: A Framework for Image and Manifold Processing. IEEE Transactions on Image Processing, 17(7), 2008.

[21] A. Elmoataz, M. Toutain, and D. Tenbrinck. On the $p$-Laplacian and $\infty$-Laplacian on graphs with applications in image and data processing. SIAM Journal on Imaging Sciences, 8(4):24122451, 2015.

[22] G. Facciolo, P. Arias, V. Caselles, and G. Sapiro. Exemplar-based interpolation of sparsely sampled images. In D. Cremers, Y. Boykov, A. Blake, and F. R. Schmidt, editors, Energy Minimization Methods in Computer Vision and Pattern Recognition, pages 331-344, Berlin, Heidelberg, 2009. Springer Berlin Heidelberg. 
[23] M. J. Fadili and G. Peyré. Total variation projection with first order schemes. IEEE Transactions on Image Processing, 20(3):657-669, 2010.

[24] K. J. Falconer. Fractal geometry : mathematical foundations and applications. J. Wiley \& sons, Chichester, New York, Weinheim, 1990. Réimpr. en 1993, 1995, 1997, 1999, 2000.

[25] N. García Trillos and D. Slepčev. Continuum limit of total variation on point clouds. Archive for Rational Mechanics and Analysis, 220(1):193-241, 2016.

[26] Y. V. Gennip and A. L. Bertozzi. Gamma-convergence of graph ginzburg-landau functionals. Advances in Differential Equations, 17:1115-1180, 042012.

[27] G. Gilboa and S. Osher. Nonlocal linear image regularization and supervised segmentation. SIAM J. Multiscale Modeling and Simulation, 6(5), 2007.

[28] G. Gilboa and S. Osher. Nonlocal operators with applications to image processing. SIAM J. Multiscale Modeling and Simulation, 7(1):1005-1028, 2008.

[29] Y. Hafiene, J. Fadili, C. Chesneau, and A. Elmoataz. Continuum limit of the nonlocal $p$ laplacian evolution problem on random inhomogeneous graphs. arXiv:1805.01754, 2018.

[30] Y. Hafiene, J. Fadili, and A. Elmoataz. Nonlocal p-laplacian evolution problems on graphs. SIAM Journal on Numerical Analysis, 56(2):1064-1090, 2018.

[31] M. Hein. Uniform convergence of adaptive graph-based regularization. In International Conference on Computational Learning Theory, pages 50-64, 2006.

[32] M. Hein, J.-Y. Audibert, and U. von Luxburg. From graphs to manifolds - weak and strong pointwise consistency of graph laplacians. In 18th Annual Conference on Learning Theory, COLT, pages 470-485. Springer, 2005.

[33] N. Kusolitsch. Why the theorem of Scheffé should be rather called a theorem of Riesz. Periodica Mathematica Hungarica, 61(1):225-229, 2010.

[34] L. Lovász and B. Szegedy. Limits of dense graph sequences. Journal of Combinatorial Theory, Series B, 96(6):933 - 957, 2006.

[35] L. Lovász and B. Szegedy. Limits of dense graph sequences. Journal of Combinatorial Theory, Series B, 96(6):933 - 957, 2006.

[36] U. V. Luxburg. A tutorial on spectral clustering. Statistics and Computing, 17(4):395-416, 2007.

[37] G. S. Medvedev. The nonlinear heat equation on dense graphs. SIAM J. on Mathematical Analysis, 46(4):2743-2766, 2014.

[38] Y. Nesterov. A method for solving the convex programming problem with convergence rate O(1/k $k^{2}$. Dokl. Akad. Nauk SSSR, 269(3):543-547, 1983.

[39] E. Pardoux. Cours intégration et probabilité. Lecture notes (Aix-Marseille Universtité), November 2009. 
[40] G. Peyré. Image processing with nonlocal spectral bases. SIAM J. Multiscale Modeling and Simulation, 7(2):703-730, 2008.

[41] G. Peyré, S. Bougleux, and L. Cohen. Non-local regularization of inverse problems. Inverse Problems and Imaging, 5(1930):511, 2011.

[42] O. Scherzer, M. Grasmair, H. Grossauer, M. Haltmeier, and F. Lenzen. Variational methods in imaging, volume 167. Springer, 2009.

[43] A. Singer. From graph to manifold laplacian: The convergence rate. Applied and Computational Harmonic Analysis, 21(1):128-134, 2006.

[44] A. Singer and H.-T. Wu. Spectral convergence of the connection laplacian from random samples. Information and Inference: A Journal of the IMA, 6(1):58-123, 2017.

[45] D. Slepčev and M. Thorpe. Analysis of $p$-laplacian regularization in semi-supervised learning. CoRR, 2017.

[46] S. M. Smith and J. M. Brady. Susan - a new approach to low level image processing. International Journal of Computer Vision, 23(1):45-78, May 1997.

[47] A. Spira, R. Kimmel, and N. Sochen. A short- time beltrami kernel for smoothing images and manifolds. IEEE Transactions on Image Processing, 16(6):1628-1636, June 2007.

[48] S. O. Stefan Kindermann and P. W. Jones. Deblurring and Denoising of Images by Nonlocal Functionals. SIAM Multiscale Modeling and Simulations, 4:25, 2006.

[49] A. Szlam, M. Maggioni, and R. Coifman. A general framework for adaptive regularization based on diffusion processes on graphs. Journal of Machine Learning Research, 19:1711-1739, 2008 .

[50] D. Ting, L. Huang, and M. I. Jordan. An analysis of the convergence of graph laplacians. In Proceedings of the 27th International Conference on Machine Learning, 2010.

[51] C. Tomasi and R. Manduchi. Bilateral filtering for gray and color images. In Sixth International Conference on Computer Vision (IEEE Cat. No.98CH36271), pages 839-846, Jan 1998.

[52] S. Vaiter, G. Peyré, and M. J. Fadili. Low complexity regularization of linear inverse problems. In G. Pfander, editor, Sampling Theory, a Renaissance, Applied and Numerical Harmonic Analysis (ANHA). Birkhäuser/Springer, 2015.

[53] U. von Luxburg, M. Belkin, and O. Bousquet. Consistency of spectral clustering. The Annals of Statistics, 36(2):555-586, 2008.

[54] J. Wang and B. J. Lucier. Error bounds for finite-difference methods for rudin-osher-fatemi image smoothing error Bounds for Finite-Difference Methods for Rudin-Osher-Fatemi Image Smoothing. SIAM Journal on Numerical Analysis, 49, 2011.

[55] Z. Yang and M. Jacob. Nonlocal regularization of inverse problems: A unified variational framework. IEEE Transactions on Image Processing, 22(8):3192-3203, Aug 2013. 
[56] L. P. Yaroslavsky. Digital Picture Processing - an Introduction. Springer, Berlin, 1985.

[57] D. Zhou and B. Schölkopf. Regularization on discrete spaces. In Proceedings of the 27th DAGM Conference on Pattern Recognition, pages 361-368. Springer-Verlag, 2005. 